\title{
Contact Detection for Nanomanipulation in Scanning Electron Microscope
}

\author{
by
}

Steve To

A thesis submitted in conformity with the requirements for the degree of Master of Applied Science

Graduate Department of Electrical and Computer Engineering

University of Toronto 


\title{
Contact Detection for Nanomanipulation in Scanning Electron
}

\section{Microscope}

\author{
Steve To \\ Master of Applied Science \\ Graduate Department of Electrical and Computer Engineering \\ University of Toronto
}

2011

\section{Abstract}

A major difficulty in the fabrication of nanostructure based electronics is the lack of effective processes capable of precisely arranging nanostructures into predefined positions. Top-down approaches introduce increased complexity and a high cost for practical industrial use, while bottom-up approaches are probabilistic in nature and do not provide precise control of nanostructure properties (i.e., number, diameter), which influence device performance. Alternatively, nanomanipulation promises specificity, precision and programmed motion and its automation may facilitate the large-scale fabrication of nanostructure based devices.

This study focuses on the development of an automated contact detection algorithm which positions an end-effector in contact with a target surface without the need for additional equipment, devices or sensors. We demonstrate this algorithm as an enabling feature for automated nano-FET biosensor construction with precise control over nanowire parameters thereby reducing device-to-device variability and also potentially allowing us to optimize individual device performance. 


\section{Acknowledgments}

I would like to express my deep thanks and appreciation to my advisor, Professor $\mathrm{Yu}$ Sun, for his guidance, support and understanding throughout my Master's studies. I am truly thankful for being given the opportunity to do work in an exciting field.

I am grateful to my colleagues at the Advanced Micro and Nanosystems Laboratory for providing a supportive and stimulating environment throughout my research. I would like to thank Yanliang Zhang for his suggestions and guidance during my studies. His experience and breadth of knowledge helped me bring this project to fruition. Thank you to Jason Li for his collaboration in the automated construction of bio-sensor devices. His development of nanowire field-effect transistor devices allowed me the opportunity to integrate my research into a significant biological application. I would also like to thank Yong Zhang, Brandon Chen, Jian Chen, and Changhai Ru for their helping hand throughout my Masters research. I am grateful for your discussion and encouragement throughout my time here.

I want to give a special thanks to Valerie, my love and best friend, whose care, companionship and enduring support helped through the hardships of life and my research. Your smile and sense of humor have always been a light in my life.

Finally, I would like to express my deep appreciation to my mother, father and little sister. Thank you for providing for me and giving me the opportunity to have so many open paths in life. Thank you for always being there for me and for believing in me. Without you, I would not be the person I am today. 


\section{Table of Contents}

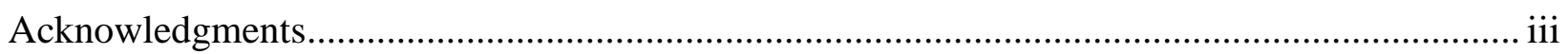

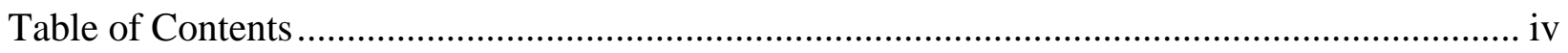

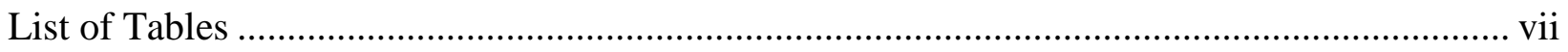

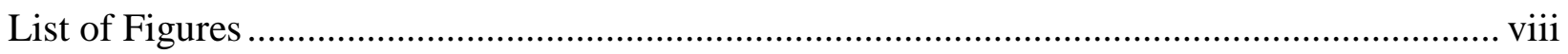

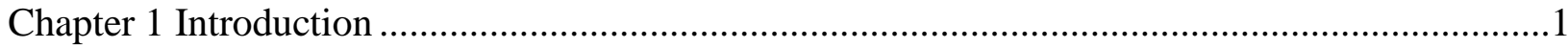

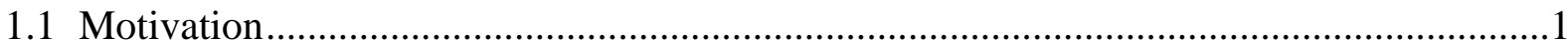

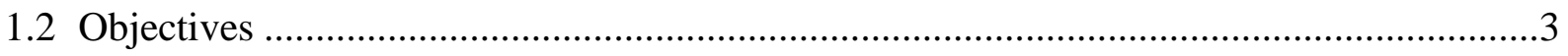

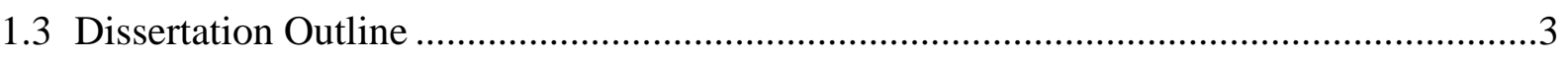

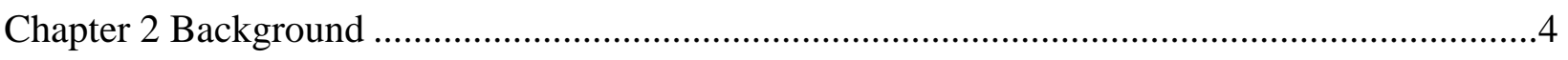

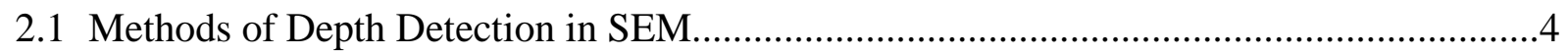

2.1.1 Integration of Additional Vision Systems ..............................................................4

2.1.2 Contact Sensor Equipped Manipulation Tools ……………...................................5

2.1.3 Computer Vision Based Depth Detection............................................................8

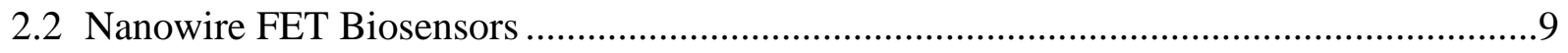

2.3 Methods for Large-Scale Nano-FET Fabrication .........................................................10

2.3.1 Bottom-Up Fabrication Methods ………………….............................................11

2.3.2 Top-Down Fabrication Method ........................................................................15

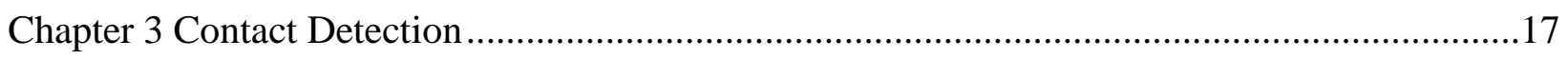

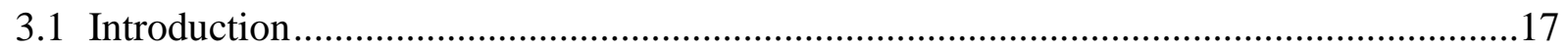

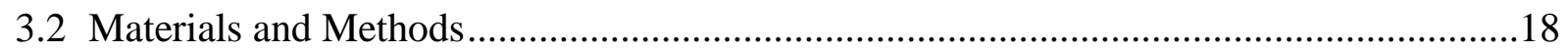

3.2.1 Nanomanipulation System .................................................................................18

3.2.2 Visual Tracking ...................................................................................19

3.2.3 Vertical Depth Estimation - Depth From Focus...................................................20 


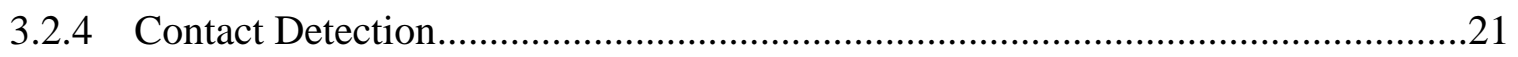

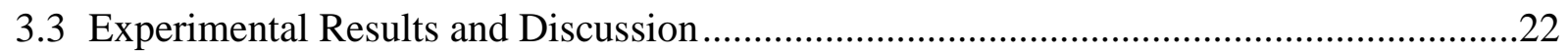

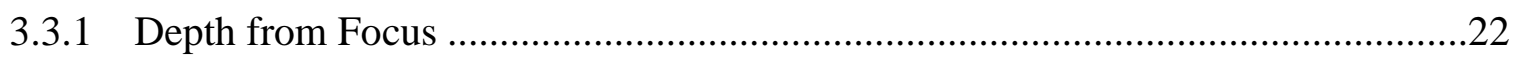

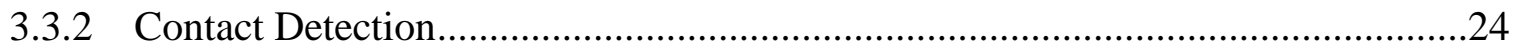

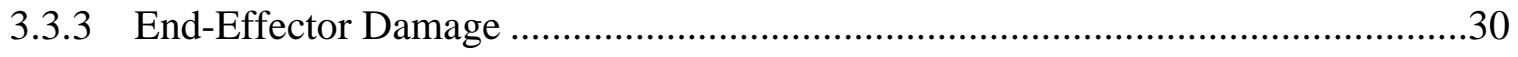

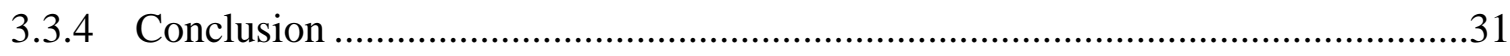

Chapter 4 Contact Detection in Nano Device Construction through Automated Nanomanipulation ..........................................................................................................

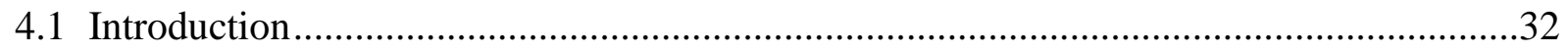

4.2 System and Device Batch Microfabrication ………….......................................................

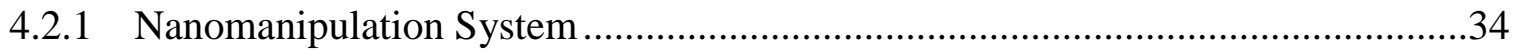

4.2.2 Device Batch Microfabrication ............................................................................36

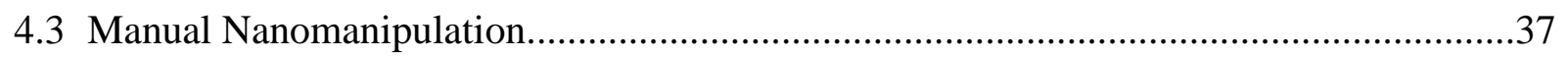

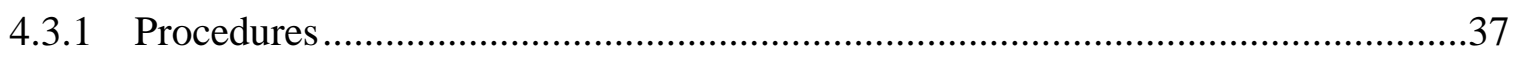

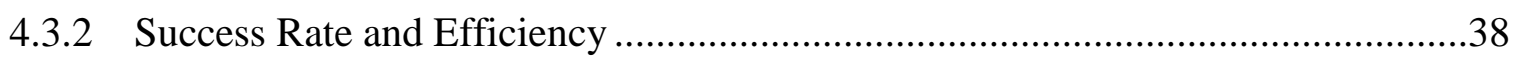

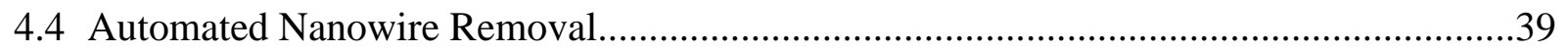

4.4.1 Automated Post Processing ......................................................................................39

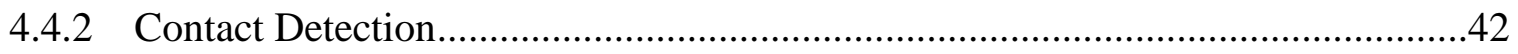

4.4.3 Nanowire Removal ............................................................................................42

4.4.4 Incorporation of Sensors into Microfluidic Device ……………............................4

4.4.5 Protein Sensing Experiments ..........................................................................4

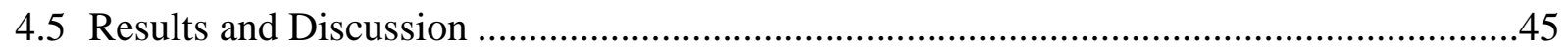

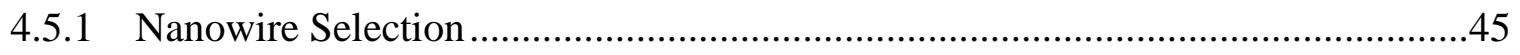

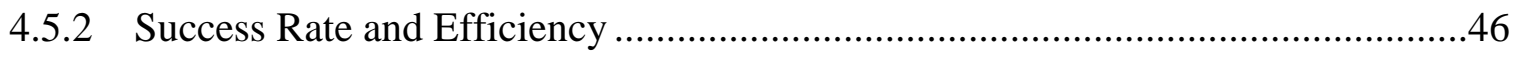

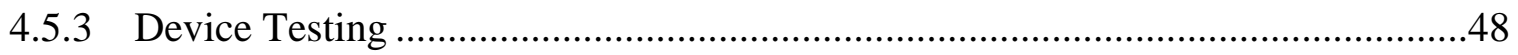

4.5.4 Varying Number and Diameter of Nanowires ..................................................50 
4.5.5 Effect of Nanowire Number.

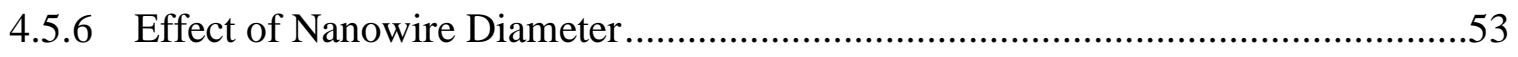

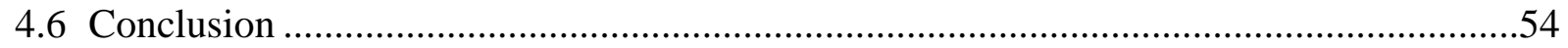

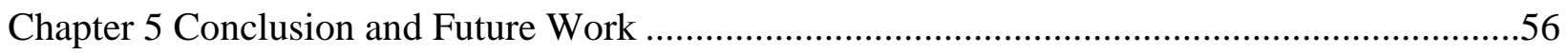

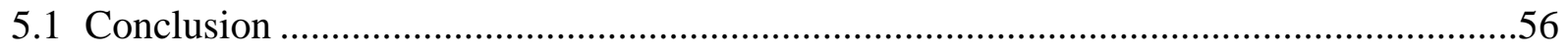

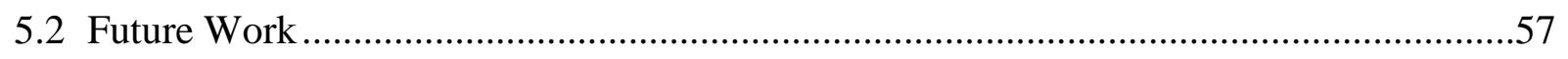

5.2.1 Contact Detection using Nanogrippers ...................................................57

5.2.2 Investigate the Effects of Nanowire Spacing in Biosensing ...............................57

5.2.3 Expand on Automated Nanomanipulation Applications ....................................58

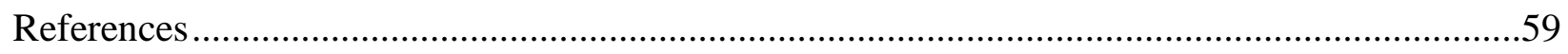




\section{List of Tables}

Table 3.1: Contact detection accuracy and deflection distance at varying magnifications.

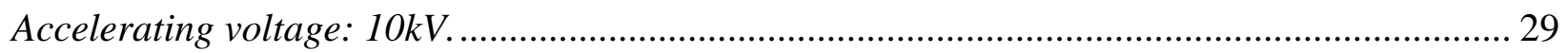

Table 3.2: Contact detection accuracy and deflection distance at varying accelerating voltages.

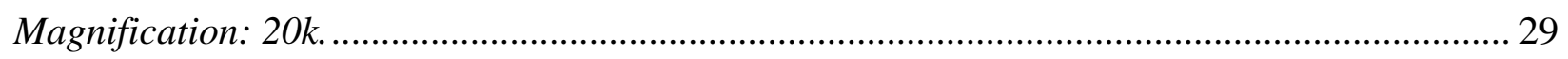

Table 4.1: Manual nanomanipulation for post processing of 60 devices .................................. 39

Table 4.2: Effect of $\Delta s_{\text {min }}$ value on number of qualified $N W s$ within each device...................... 45

Table 4.3: Effect of $\Delta s_{\text {min }}$ value on failure rate of $N W$ severing ............................................. 46

Table 4.4: Overall performance of nanorobotic manipulation................................................. 47 


\section{List of Figures}

Figure 2.1: Approach of the micro gripper to the micro object. The relative distance of the probe to the substrate surface is determined by the reflective scale. (Adapted with permission from [36]

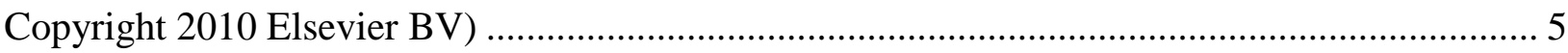

Figure 2.2: Touch sensor with vibrating piezoelectric element. The sensor has two electrodes: one for driving one for sensing. Mechanical impedance changes when in contact with an object. (Adapted with permission from [40] Copyright 2006 Elsevier Science)

Figure 2.3: MEMS-based microgripper with integrated two-axis capacitive force sensors. (Adapted with permission from [41] Copyright 2009 Sage Science Press (UK)) 7

Figure 2.4: A single nano-FET biosensor. Charged analyte material links to the functionalized nanostructure, thereby inducing a measurable change in conductance. 10

Figure 2.5: Schematic of fluidic channel structures for flow assembly. Fluid flow is used to align suspended nanowires onto a substrate. (Adapted with permission from [49] Copyright 2001 American Association for the Advancement of Science).

Figure 2.6: Contact printing of nanowires. (a) Schematic of the contact printing process for producing well-aligned nanowire arrays. (b) Dark-field optical and (c) SEM images of $30 \mathrm{~nm}$ nanowires printed on a $\mathrm{Si} / \mathrm{SiO} 2$ substrate showing highly dense and aligned monolayer of nanowires. The self-limiting process limits the transfer of nanowires to a single layer, without significant nanowire bundling. (Adapted with permission from [50]. Copyright 2008 American Chemical Society.) 
Figure 2.7: Linker-free directed self-assembly. (a) The patterning process of octadecyltrichlorosilane self-assembled monolayer on a solid substrate via photolithography. (b) Self assembly suspended nanostructures onto the bare surface regions on the substrate. Subsequent photolithography and metallization can be used to pattern electrodes onto the ends of the deposited nanostructures. (Adapted with permission from [52] Copyright 2006 Nature Publishing Group) 14

Figure 2.8: (a) Schematic of Nano-FET device after anisotropic etching. The silicon-on-insulator active channel (yellow, width w and thickness t) is undercut etched, whereas degerate leads (red) are etch-resistant. The source (S), drain (D), and underlying backgate (G) are labeled. (b) Scanneing electron micrograph of a complete device. (Adapted with permission from [22] Copyright 2007 Nature Publishing Group) 16

Figure 3.1: Nanomanipulators equipped with nanoprobes inside SEM. 18

Figure 3.2: Nanoprobe tip is visually tracked using normalized cross correlation template matching. $T(x, y)$ is the template image. A search window within the proximity of the probe tip is chosen as the ROI, $I(x, y)$ 19

Figure 3.3: Probe slides on the substrate after contact is established, which translates to horizontal motion in the image plane. Electrical connections to the probe and substrate are for electrical verification of contact. 22

Figure 3.4: Normalized variance (NV) changes as the probe descends at $100 \mathrm{~nm}$ per image frame at $10 \mathrm{k}$ magnification and $5 \mathrm{kV}$ accelerating voltage. Between $A$ and $B$, the probe is within the depth of field (DOF). Slope is used to determine when the probe is within DOF. The probe stops 
when the normalized variance slope reaches a threshold of $k \sigma$ where $\sigma$ is the standard deviation of the slope and $k$ is a positive integer. 23

Figure 3.5: Contact detection and subsequent $Z$ positioning of a probe via sub-pixel normalized cross correlation tracking (SEM: $10 \mathrm{kV}$ accelerating voltage, 10k magnification; motion step size produced by nanomanipulator: $10 \mathrm{~nm}$ ). Electrical current measurement is used to verify contact between the probe tip and conductive substrate. 25

Figure 3.6: Quantification of contact detection accuracy. (a) Probe is initially positioned above the substrate at a distance, $z_{a}$, after vision based contact detection is completed. (b) Probe moves laterally towards nanosphere to establish contact which is verified via electrical current monitoring. (c) Probe is raised by one step, $Z_{s}$ and laterally moved again to touch the nanosphere. (d) This process is repeated until the probe is raised high enough, and no longer comes in contact with the nanosphere. (e)(f) SEM images captured in the probe nanosphere contact experiments. Nanosphere was $100 \mathrm{~nm}$ in diameter. 27

Figure 3.7: Damaged nanoprobe resulting from manual manipulation. 30

Figure 3.8: Measurement of permanent deformation of the probe tip before and after 200 times contact detection. 31

Figure 4.1: Schematic of nano-FET sensors for sensing hIgG protein. Nanowire diameter and number vary across devices, resulting in sensitivity variations. 33

Figure 4.2: (a) Nanomanipulation system. 1. nanomanipulators-1, 2. nanomanipulators-2, 3. nanoprobe, 4. Batch fabricated nano-FET array mounted on the SEM stage for nanomanipulation. (b) The system is mounted onto and demounted from the SEM through the 
specimen exchange chamber. (c) SEM image of 8 nano-FET devices where microelectrodes are on top of the nanowires and pin the nanowires to the substrate. 35

Figure 4.3: Physical removal of selected nanowires. (a) Operator decides which nanowire(s) to keep. (b) Lower nanoprobes to the substrate surface. (c) Sever undesired nanowires. (d) Perform electrical characterization. 37

Figure 4.4: (a) Diameter and distances to adjacent nanowires are determined for each nanowire. (b) A device image showing electrode bridging nanowires (electrodes not shown in image). (c) Nanowire contours are extracted from image processing. 41

Figure 4.5: Look-then-move and closed-loop position control. 43

Figure 4.6: (a)(b) Before and after nanomanipulation removal of nanowires between source and drain electrodes. (c) An array of 4 single-nanowire devices (arrows: remaining desired nanowire). Inset shows a high-resolution SEM image of a target nanowire. 47

Figure 4.7: Current-voltage characteristics of a post-processed nanowire FET device $\left(\mathrm{V}_{\mathrm{sd}}=0\right.$ to $20 \mathrm{~V}, \mathrm{~V}_{\text {backgate }}=-25$ to $+25 \mathrm{~V}$, increasing $\mathrm{V}_{\text {backgate }}$ indicated by arrow). 49

Figure 4.8: A typical current-time response for 7 Si-nanowire FET biosensors exposed to increasing concentrations of hIgG in $0.1 x$ PBS buffer (from $10 \mathrm{fg} / \mathrm{mL}$ to $10 \mu \mathrm{L}$ ). 50

Figure 4.9: Effect of nanowire number on device sensitivity and variability. (a) Normalized current as a function of protein concentration for devices with 1,4 and 7 nanowires $(\mathrm{n}=4$ for each group; nanowire diameter 80-100 nm; nanowire doping density $=10^{19}$ atoms $\cdot \mathrm{cm}^{-3}$ ). 
Sensitivity as a function of the number of bridging nanowires ( $\mathrm{n}=4$ for each group). Decreasing sensitivity was observed for higher numbers of nanowires. $(* \mathrm{p}<0.05)$ 52

Figure 4.10: Effect of nanowire diameter on device sensitivity. Single-nanowire devices with mean diameters of 60-80 nm, 81-100 nm, and 101-120 nm were characterized. (a) Normalized current as a function of protein concentration for single-nanowire devices with different nanowire diameters ( $\mathrm{n}=6$ for each group, doping concentration of $10^{17}$ ). (b) Smaller nanowire diameters produced more sensitive nanowire FET biosensors. $\left(\mathrm{n}=6\right.$ for each group; $\left.{ }^{*} \mathrm{p}<0.05\right) . .54$ 


\section{Chapter 1 Introduction}

\subsection{Motivation}

Nanostructures have been demonstrated in numerous device applications ranging from AFM imaging[1] and photodetection [2] to chemical [3-6] and biological [7-11] sensing. These devices utilize the unique properties of nanowires, nanotubes, nanoribbons, etc., as their active element. While device prototyping is possible through existing techniques, commercial-scale fabrication of nanodevices with high throughput and minimal device-to-device variability is difficult. One of the major challenges in the fabrication of nanodevices is the incorporation of nanostructures into predefined orientation and patterns.

Top-down micro- and nano-fabrication of nanostructure devices utilize well-known processes such as electron-beam nanolithography followed by dry etching [7], silicon wire thinning via repeated surface oxidation and HF etching processes $[12,13]$ and anisotropic timed etching of silicon structures to nanoscale feature sizes [14]. These top-down techniques involve high processing costs/complexity, low yields associated with e-beam lithography and focused ion beam (FIB), and significant variability across etched devices due to etching non-uniformity across the wafer and its high sensitivity to processing conditions.

Alternatively, pre-synthesized nanostructures (i.e. nanotubes and nanowires) are of low cost and can be integrated into micro-structures to form nanodevices. Existing wafer-scale nanotube/nanowire integration methods, such as directed assembly [15], contact printing [16], and dielectrophoresis [17] are inexpensive; however, they are incapable of precisely controlling

nanostructure parameters, such as the number nanostructures and nanostructure size, which 
influence device performance [18-25]. This results in a large device-to-device variability as their performance is mainly dependent on these nanostructure parameters.

By contrast, mechanical nanomanipulation, despite being serial in nature and slower compared to abovementioned wafer-scale nanostructure integration methods, promises specificity, precision, and programmed motion. In addition, using a vision sensor allows us to selectively manipulate nanostructures based on their characteristics (i.e., number, diameter, etc.). A combined use of wafer-scale processes and automated nanomanipulation would constitute a new paradigm to bring nanomanufacturing one step further towards scaled-up operation and in the meanwhile, achieve high precision control of nanostructure parameters.

Nanomanipulation inside scanning electron microscope (SEM) [26-31] has been largely performed manually by an operator. Manual operation requires the operator to carefully monitor the SEM screen and operate joysticks to control the positioning of nanomanipulators making it time-consuming, skill-dependent and has low productivity. Since nanomanipulation is a 3dimensional task, accurate knowledge in all three spatial coordinates is necessary. SEM images deliver high resolution $2 \mathrm{D}$ information enabling the automated planar positioning of nanorobotic manipulators $[32,33]$; however, the lack of depth information impedes the most crucial and time consuming part of nanomanipulation, the approach of the end-effector in the z-direction. Therefore, automated contact detection, whereby the end-effector is positioned in contact with a target surface, must be implemented to facilitate the nanomanipulation of nanostructures.

Hence, for commercial-scale fabrication of nanostructure devices a method utilizing wafer-scale processes for high throughput in combination with contact detection and automated 
nanomanipulation to precisely control nanostructure parameters thereby achieving desired device performance and subsequently, reducing device-to-device variability.

\subsection{Objectives}

The objective of this thesis is to develop a contact detection algorithm to facilitate automated nanomanipulation of nanostructures. We also aim to integrate automated contact detection in a nanodevice fabrication process using both wafer-scale methods and nanomanipulation to allow precise control of nanostructure parameters. Specifically, we want to

1.) Develop an automated contact detection algorithm which positions an end-effector in contact with a target surface with no additional equipment, devices or sensors.

2.) Utilize the contact detection algorithm for the post processing of nanodevices, specifically nanowire field-effect transistor (nano-FET) biosensors with improved control over nanowire parameters (i.e., nanowire number and diameter), thereby reducing deviceto-device variability, which translates to minimized performance variability.

\subsection{Dissertation Outline}

An overview of the ensuing chapters is as follows: Chapter 2 provides a literature review of past methods of obtaining depth information within micro/nanomanipulation systems. We then provide an overview of biosensor functionality including existing fabrication methods that position nanostructures onto devices. Chapter 3 presents and characterizes our automated visionbased contact detection algorithm for the approach of an end-effector to a target surface. Chapter 4 describes the fabrication of nano-FET sensors with carefully controlled device parameters and reduced variability through contact detection and automated nanomanipulation. Finally, Chapter 5 summarizes the contributions of this research and suggested future research directions. 


\section{Chapter 2 Background}

\subsection{Methods of Depth Detection in SEM}

Obtaining depth information is especially important in automated nanomanipulation as much care is needed during the approach of the end-effector to the target object/surface. In addition to accurately approaching the target object, the end-effector must also avoid hard collisions with other objects to prevent end-effector damage. Existing methods of obtaining depth information in SEM have relied on the integration of additional vision systems, contact sensor equipped end-effectors, or computer vision based methods.

\subsubsection{Integration of Additional Vision Systems}

Earlier methods of obtaining depth information involved mounting optical microscopes to the side of the SEM sample chamber [34]. In this configuration, XY-positioning information is provided by SEM imaging while Z-positioning feedback is provided by the optical microscope, however, the need for additional hardware and the small depth of focus in optical microscopy complicates the nanomanipulation process. In addition, the resolution is limited to $2 \mu \mathrm{m}$, which is insufficient for nanomanipulation.

Laser triangulation systems have also been implemented in SEM manipulation systems [35]. In this configuration, the laser shoots a sheet of light onto the target substrate and a miniaturized light optical microscope is used for depth measurement using laser triangulation inside SEM. Although this method is capable of accurate microscopic manipulation, the limitations of the optical microscope and the thickness of the laser sheet prevent it from being used on the nano-scale. The brightness of the laser is also material dependent as dark and 
transparent materials cannot be detected on the object. Brightness is also affected by the operating conditions of the SEM, making automation processes difficult to implement.

Recently, the use of an infrared camera in conjunction with a reflective micro-patterned scale installed perpendicular to a reflective substrate was reported, as shown in Figure 2.1 [36]. By monitoring the end-effectors reflection on the substrate in conjunction with the end-effectors position relative to the scale, the position between the end-effector and the target object can be determined. However, poor scale resolution and low magnification of light microscopy provided poor accuracy (worse than $5 \mu \mathrm{m}$ ).

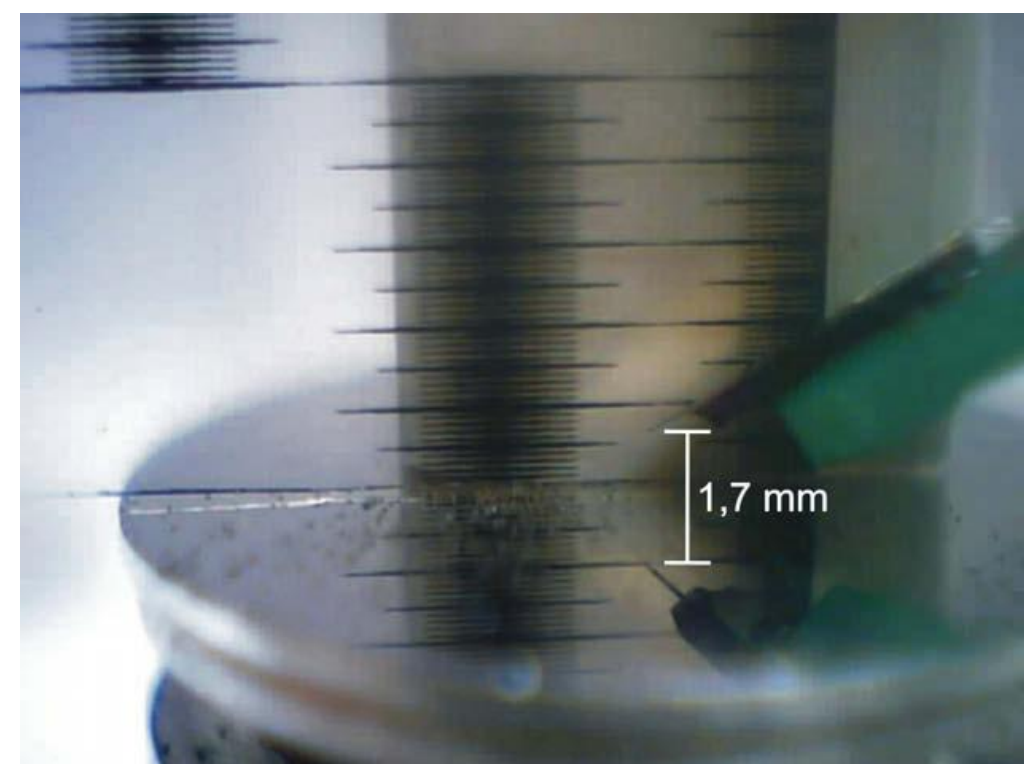

Figure 2.1: Approach of the micro gripper to the micro object. The relative distance of the probe to the substrate surface is determined by the reflective scale. (Adapted with permission from [36] Copyright 2010 Elsevier BV)

\subsubsection{Contact Sensor Equipped Manipulation Tools}

Touch sensors (piezoresistive [37, 38], piezoelectric [33, 39, 40], and capacitive[41]) have also been used for contact detection. Piezoresistive and piezoelectric sensors take advantage 
of special materials whose properties are changed in response to an applied mechanical stress. Capacitive sensors make use of changes in capacitance experienced when the distance between capacitor plates is changed. By integrating these materials into end-effectors, contact can be detected by monitoring the change in these properties.

Piezoresistive touch sensors are used in contact detection by monitoring resistance changes that occur when a mechanical force is applied to the sensing material [37, 38]. By integrating piezoresistive materials into end-effectors, contact can be established by monitoring resistance changes of the piezoresistors as the end-effector undergoes mechanical stress from end-effector deformation when contacting the substrate.

Similarly, piezoelectric touch sensors detect contact by sensing mechanical impedance changes that occur when the end-effector collides with the substrate [39, 40]. In the design shown in Figure 2.2, the touch sensor uses two piezoelectric elements: one for sensing, and one for driving vibration at a resonant frequency. As the sensor vibrates and contacts a target surface, the mechanical impedance change is detected by the sensing voltage phase and/or amplitude changes.

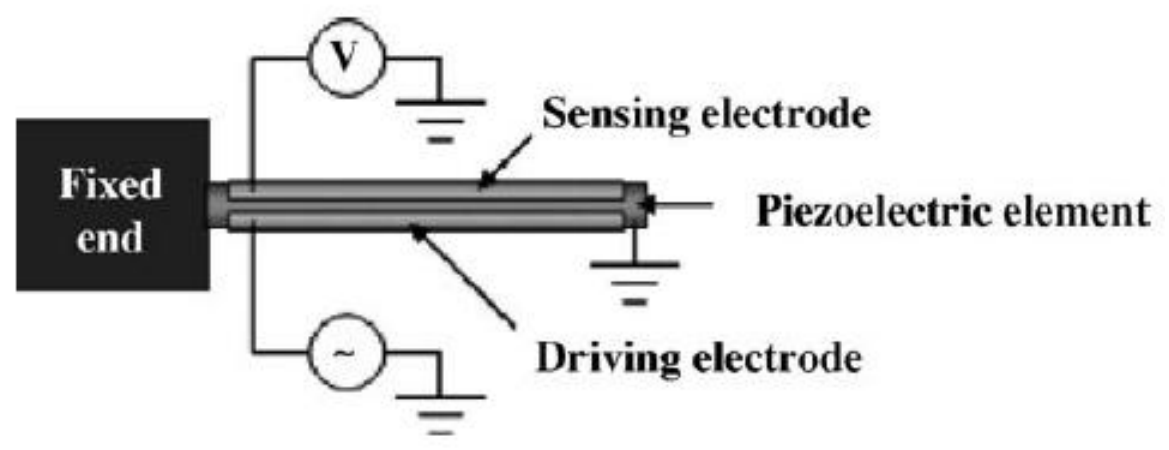

Figure 2.2: Touch sensor with vibrating piezoelectric element. The sensor has two electrodes: one for driving one for sensing. Mechanical impedance changes when in 
contact with an object. (Adapted with permission from [40] Copyright 2006 Elsevier Science)

Microgrippers equipped with two-axis capacitive comb force sensors, as shown in Figure 2.3, are capable of both providing gripping force feedback and contact sensing[41]. The capacitive combs comprise of a single plate fixed to the bulk of the device with the other attached to the microgripping arms. When the gripper comes into contact with the substrate, the force will cause a displacement in parallel plates effectively changing the capacitor plate gap, which in turn changes the capacitance. By monitoring changes in capacitance, contact detection is achieved.

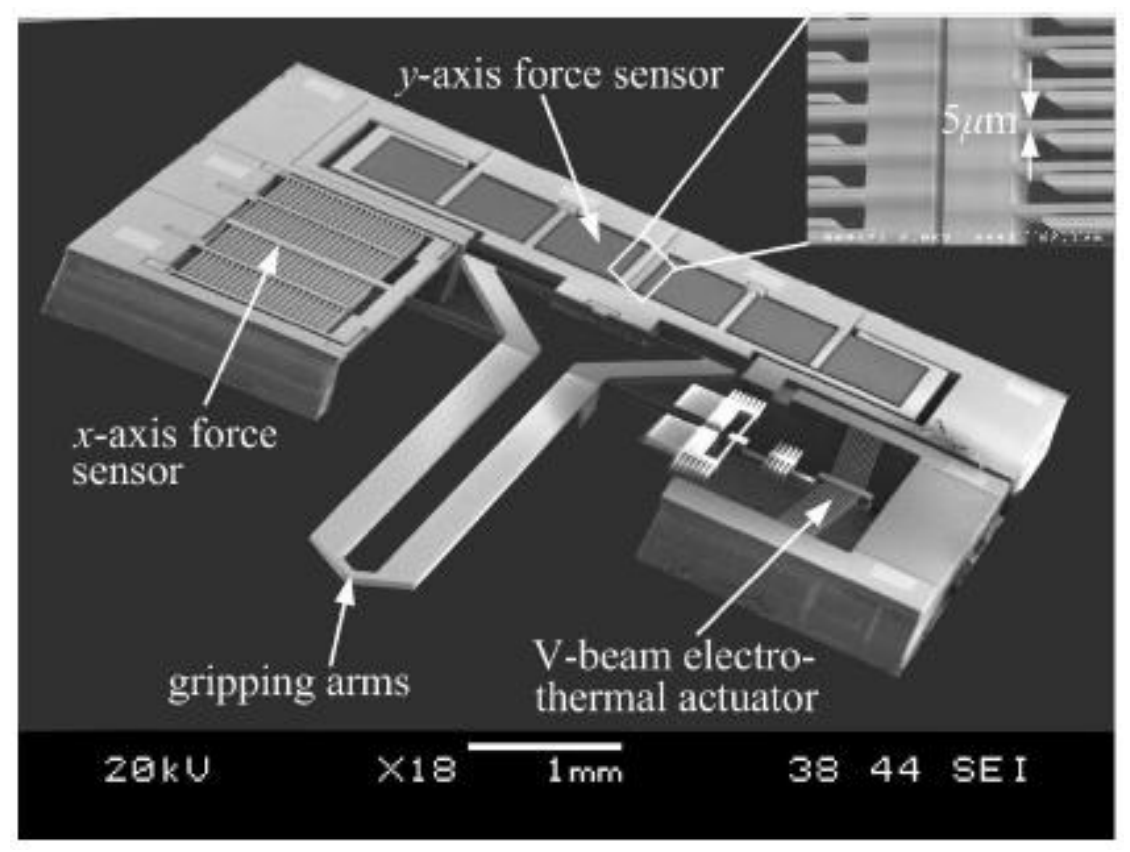

Figure 2.3: MEMS-based microgripper with integrated two-axis capacitive force sensors. (Adapted with permission from [41] Copyright 2009 Sage Science Press (UK))

Although these MEMS based sensors are capable of contact detection, the integration of these sensors into a nanomanipulation system is difficult (e.g., via epoxy). Furthermore, as 
contact sensors at micro-nano-Newton levels are fragile and highly prone to damage, extra care must be taken in the process of integrating these devices as well as in the process of nanomanipulation.

\subsubsection{Computer Vision Based Depth Detection}

Three-dimensional visual information can also be obtained by analyzing stereoscopic SEM images gathered by applying rotational and translational changes to the specimen stage [42]. The major disadvantage of this approach is that the sample, nanomanipulator, and endeffector must all be rotated/translated. Since the tilting axis of the sample inside SEM is underneath the sample plane, the sample disappears from the electron beam and thus out of the field of view. The stage must then be translated to position the sample within the field of view, slowing down and bringing complexity to the nanomanipulation process. In addition to this, through axis tilting, the sample is effectively positioned either closer to, or further from the SEM detector, resulting in more or fewer electrons being detected, respectively. This produces images with contrast differences which may bring difficulty to processing stereoscopic images.

To circumvent the problems associated with direct sample rotation/translation, an alternative method was developed to acquire stereoscopic images through electron beam tilting [43-45]. By magnetically deflecting the beam, two stereoscopic SEM images can be obtained. However, this method requires the installation of specialized hardware into a standard SEM.

Depth-from-focus methods were also implemented in SEM nanomanipulation systems

for estimating the $\mathrm{Z}$ position of an end-effector relative to the object being manipulated [33]. This method takes advantage of the blurring of an object when the SEM is not optimally focused (not within the focal plane). By monitoring the sharpness of an end-effector relative to a target 
object within the focal plane, depth information can be obtained. Although depth-from-focus methods are often used in optical microscopy $[46,47]$, the large depth of field of the SEM makes it difficult to precisely align the end-effector tip and target object to an exact co-plane.

A shadow-based depth detection method [48] was used to vertically align a microgripper with a protruding carbon nanotube. This method takes advantage of the experimental observation that an end-effector will cast a shadow onto a surface when it is within close proximity. The performance of this shadow-based approach is highly affected by end-effector geometries and SEM imaging conditions such as accelerating voltage because higher accelerating voltages result in a deeper penetration length therefore reducing the shadowing effect.

\subsection{Nanowire FET Biosensors}

Nanoscale field effect transistor (nano-FET) biosensors are nano-scale electronic devices used to detect and measure the presence and concentration of specific biological molecules within a given sample solution. These sensors use semiconducting nanostructure (i.e. nanowire, carbon nanotube) as the transistors 'channel' bridging the source and drain electrodes, as shown in Figure 2.4. While the gate electrode in a nano-FET is typically located directly above the channel and separated by a thin dielectric layer, a nano-FET biosensor uses a solution gate electrode that is immersed within the aqueous solution being measured. 


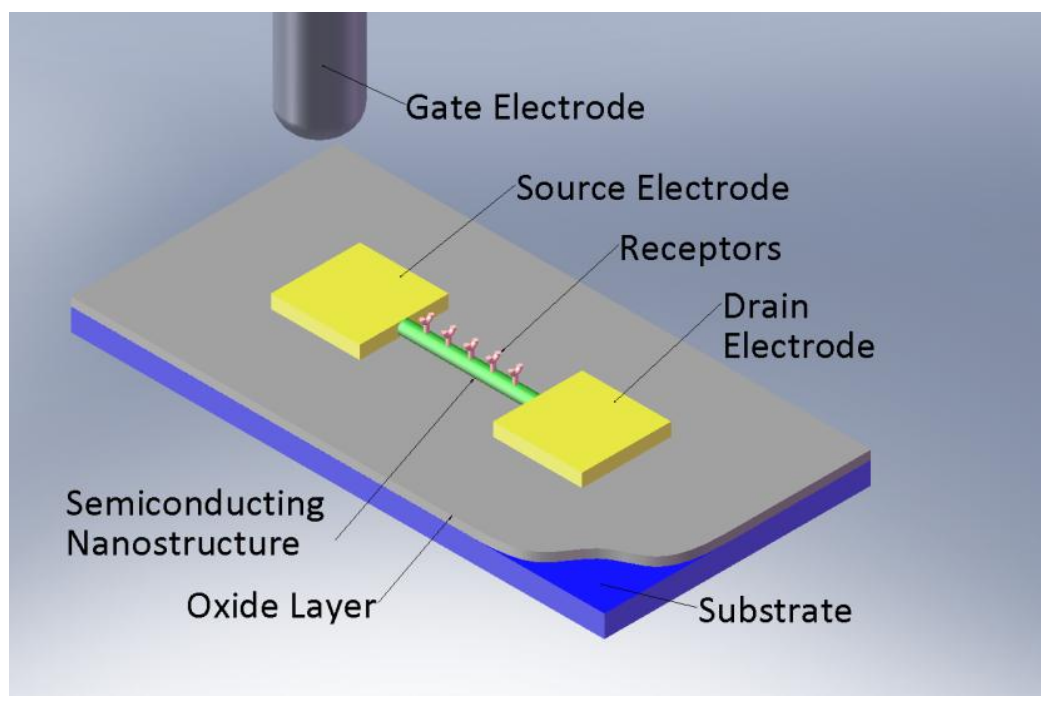

\section{Figure 2.4: A single nano-FET biosensor. Charged analyte material links to the functionalized nanostructure, thereby inducing a measurable change in conductance.}

The nanostructure surface is functionalized with a layer of molecular receptors specific to the analyte being measured. This enables specificity for the sensor as only certain molecules will link to the nanowire surface after functionalization. The presence of an analyte material is detected when the charged molecules link to the receptors, thereby modulating the number of free charge carriers within a semiconductor material which results in a measurable change of conductance within nanowire.

\subsection{Methods for Large-Scale Nano-FET Fabrication}

Early methods for manufacturing nano-FETs involve (1) using chemical vapor deposition to grow individual nanostructures, (2) transferring as-grown nanostructures from a growth substrate onto the device substrate, which results in random placement and orientation of the deposited nanostructures, and (3) the patterning of metal electrodes onto the ends of a suitable nanostructure using a mask-less nano-lithographic tool (e.g., electron beam lithography or focused ion beam lithography), which has low throughput and is only suitable for proof-of- 
principle use. Recent methods for wafer-scale production of nano-FET arrays fall into two categories: 'bottom-up' and 'top-down' fabrication methods.

\subsubsection{Bottom-Up Fabrication Methods}

A strategy for bottom-up fabrication is to use pre-synthesized free-standing nanostructures made from epitaxial growth methods (i.e., chemical vapor deposition), which offers several advantages over top-down and other bottom-up approaches including (1) the capability to assemble nano-FET devices onto flexible and transparent substrates, (2) control over the dimensions and electrical properties of the nanostructure, and (3) the ability to incorporate unique chemical compositions and architecture of nanostructures into nano-FET devices. These methods rely primarily on the transfer, alignment and positioning of as-grown nanostructures onto a device substrate. Contacting electrodes may be patterned onto the transferred nanostructures via conventional photolithography to form individual sensors or sensor arrays.

\subsubsection{Flow Alignment}

Flow alignment is a process that aligns suspended nanostructures along a single orientation using fluid flow. Nanostructures are streamed through a microfluidic structure formed between a poly(dimethtylsiloxane) mold and the device substrate, as shown in Figure 2.5 [49]. This results in nanowires assembling onto the device substrate in a relatively aligned configuration parallel to the direction of flow. The degree of alignment is a function of the flow rate as increased flow velocities result in narrower angle distribution. The density of deposited nanostructures is a result of the duration of nanostructure flow, with longer duration resulting in a denser distribution of nanostructures. This method is useful in controlling the degree of nanostructure alignment and deposition densities. Nano-FETs can then be achieved by patterning 
electrodes onto the device using conventional microfabrication processes, where the gap between electrodes dictates the number of bridging nanowires. Although a smaller gap increases the likelihood of bridging nanowires, the exact number of bridging nanowires is probabilistic.
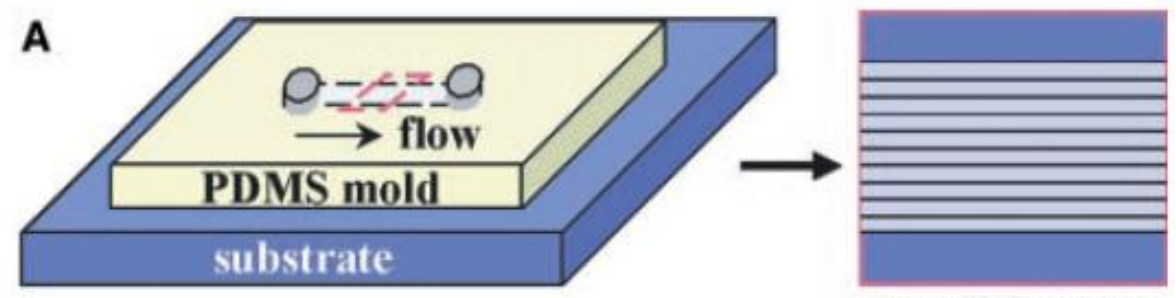

parallel array

Figure 2.5: Schematic of fluidic channel structures for flow assembly. Fluid flow is used to align suspended nanowires onto a substrate. (Adapted with permission from [49] Copyright 2001 American Association for the Advancement of Science)

\subsubsection{Nanostructure Contact Printing}

Nanostructure contact printing is similar to flow alignment, however, instead uses mechanical shear forces and friction to detach and align nanostructures from a growth substrate onto a device substrate [50]. The growth substrate is inverted and placed on top of the device substrate such that the nanostructures are sandwiched in between, as shown in Figure 2.6. Applied pressure is controlled by weights on top of the growth substrate, which guides deposited nanostructure density. The growth substrate is then pulled over the device substrate at a constant velocity to deposit and align the nanostructures onto the substrate. The shear velocity and amount of friction between the substrate influences the degree of nanostructure alignment, the latter which can be adjusted through the use of a lubricant. 

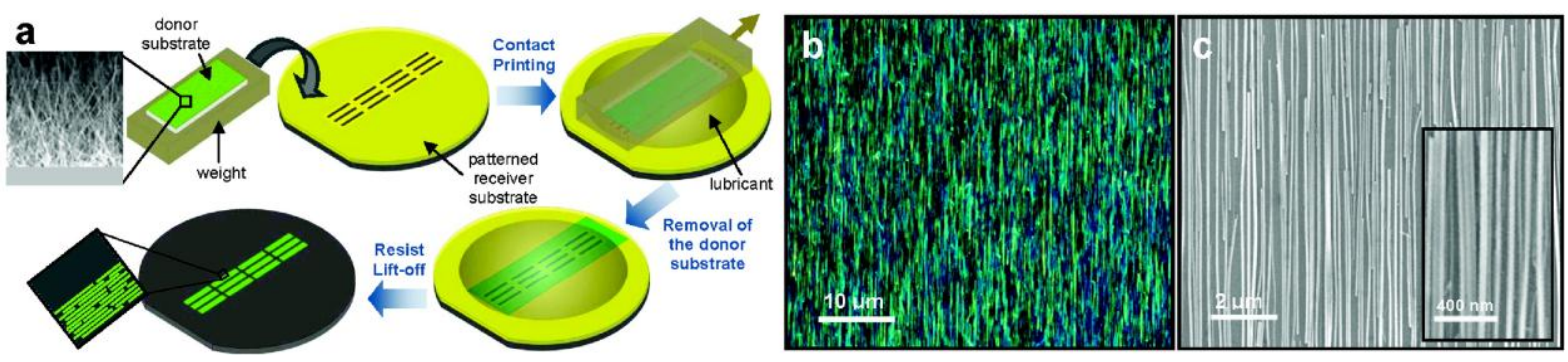

Figure 2.6: Contact printing of nanowires. (a) Schematic of the contact printing process for producing well-aligned nanowire arrays. (b) Dark-field optical and (c) SEM images of 30 nm nanowires printed on a $\mathrm{Si} / \mathrm{SiO} 2$ substrate showing highly dense and aligned monolayer of nanowires. The self-limiting process limits the transfer of nanowires to a single layer, without significant nanowire bundling. (Adapted with permission from [50]. Copyright 2008 American Chemical Society.)

\subsubsection{Thin-Film Nanowire Suspension}

Thin-film nanowire suspension is a method that makes use of the observation that suspended nanostructures suspended within a liquid thin film will align along a single direction when that film is elongated. This requires the formation of a large balloon from a nanostructure suspension [51]. The aligned nanostructures within the thin film can then be transferred to a substrate by placing the substrate in contact with the balloon. Nano-FET fabrication would subsequently proceed as in the case of flow alignment.

\subsubsection{Directed Self-Assembly}

Directed self-assembly relies on the minimization of surface energy to assemble suspended nanostructures into predefined locations on a substrate, as shown in Figure 2.7 [52]. In this method, surface modification strategies are used to render certain portions of the device substrate either hydrophobic or hydrophilic. Upon immersion of the substrate into a 
nanostructure suspension, individual nanostructures will self-assemble onto the hydrophobic portions of the substrate. This strategy can be used to deposit individual nanostructures at specific locations over a large area on a substrate. A similar strategy using analyte-ligand interactions has also been employed in which suspended nanostructures are functionalized with biotin molecules. Upon immersion of a substrate with bound streptavidin molecules at predefined locations, biotin-streptavidin conjugation forces the nanostructures to assemble onto the device substrate at pre-defined locations.
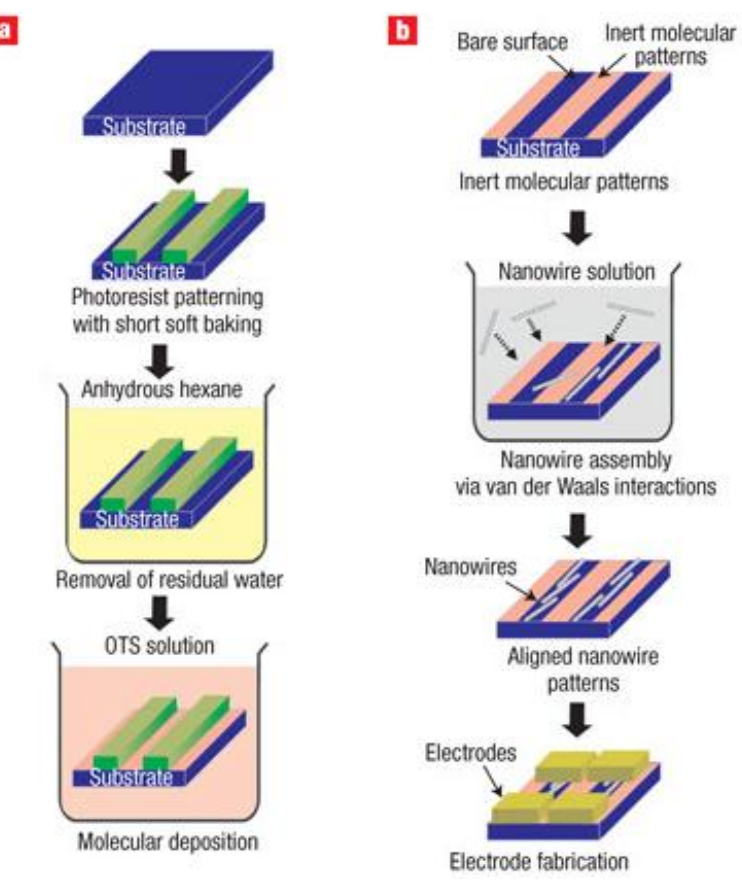

Figure 2.7: Linker-free directed self-assembly. (a) The patterning process of octadecyltrichlorosilane self-assembled monolayer on a solid substrate via photolithography. (b) Self assembly suspended nanostructures onto the bare surface regions on the substrate. Subsequent photolithography and metallization can be used to pattern electrodes onto the ends of the deposited nanostructures. (Adapted with permission from [52] Copyright 2006 Nature Publishing Group) 


\subsubsection{Dielectrophoresis}

Dielectrophoresis (DEP) utilizes electrical fields to manipulate nanostructures. In this method, metallic contact electrodes are patterned on the device substrate prior to the assembly of nanostructures onto the device. By applying a biased alternating voltage across the electrodes, a local non-uniform electric field is produced, exerting a force on suspended semi-conducting nanostructures. This force causes the nanostructures to assemble across the two electrodes. This process is easily amenable to the large-scale production of nano-FET arrays and can be used to fabricate single nanostructure FET devices, however typical DEP trapping processes yield networked multi-nanostructure FET devices.[53] This process is highly susceptible to the experimental conditions including the size, shape, and properties of the nanostructure to be manipulated, and parameters of the electrical signals, as well as the electrical properties of the surrounding medium.

\subsubsection{In-situ Growth of Nanostructures}

In-situ growth of nanostructures is a method that utilizes patterned catalysts to selectively synthesize nanostructures at certain regions on the device substrate. These catalysts (e.g., iron or gold particles) are required to initiate and/or propagate nanostructure growth. In some cases, the direction of nanostructure growth can be influenced with the application of an electric field, therefore enabling control of the resulting nanostructure orientation. This method usually produces numerous nanostructures and is therefore, useful in fabricating nano-FET sensors that utilize a network of nanostructures.

\subsubsection{Top-Down Fabrication Method}

Top-down methods are typically based on the anisotropic lateral wet etching of nanometer-thin SOI (silicon-on-insulator) wafers to produce well-defined nanostructures from 
the device layer, as shown in Figure 2.8 [22]. Micron-sized etch masks are patterned via conventional photolithography and anisotropically time-etched to produce nanometer-wide nanostructures. Source and drain electrodes are degenerately doped, rendering them conductive and unaffected by the etchant. This approach enables wafer-scale formation of semiconducting nanostructures at precise locations on a wafer-scale, making subsequent electrode fabrication relatively straightforward using conventional photolithography. As this method relies on timecontrolled anisotropic etching for the removal of material, it is highly susceptible to small changes in processing conditions such as etch time, processing temperature and mixing, and device-layer doping density and crystal orientation.
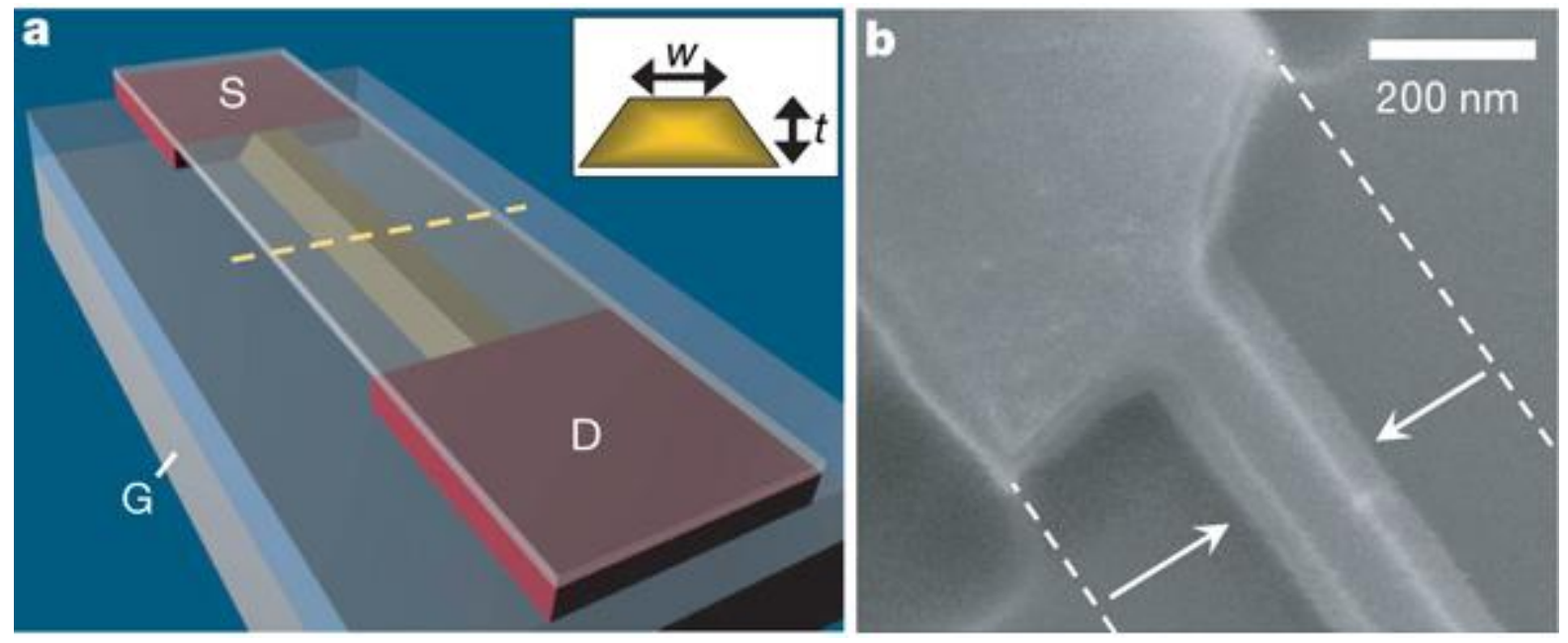

Figure 2.8: (a) Schematic of Nano-FET device after anisotropic etching. The silicon-oninsulator active channel (yellow, width w and thickness t) is undercut etched, whereas degenerate leads (red) are etch-resistant. The source (S), drain (D), and underlying backgate (G) are labeled. (b) Scanning electron micrograph of a complete device. (Adapted with permission from [22] Copyright 2007 Nature Publishing Group) 


\subsection{Introduction}

\section{Chapter 3 Contact Detection}

Accurate positioning of nanomanipulators and end-effectors is necessary nanomanipulation for the characterization of nanomaterials [31, 48, 54-58] and the guided synthesis of nanodevices [27, 59-63]. A nanomanipulation system consisting of a scanning electron microscope (SEM) and piezoelectric manipulators represents a powerful platform capable of performing simultaneous imaging and manipulation at the nanometer scale. Since nanomanipulation is a 3-dimensional task, accurate knowledge in all three spatial coordinates is necessary. Due to the lack of depth information along the $\mathrm{Z}$ direction, positioning of an endeffector (e.g., nanoprobe) vertically is based on trial and error, thus, time-consuming and skill dependent. Therefore, automated contact detection must be implemented in order for SEM nanomanipulation to move towards automation.

This chapter reports on a computer-vision based contact detection algorithm that determines the contact point between an end-effector and a target surface without the need for additional equipment, devices or sensors. Using SEM images as vision feedback, the endeffector is tracked in real-time as it descends towards the target surface using a depth from focus method. Once, the probe is within the depth of focus with the target surface, the system will transition to fine contact detection where the probe will descend at a relatively slower speed. As contact between the end-effector and target surface is established, further motion of the endeffector in the vertical $Z$ direction will translate into the end-effector sliding across the substrate plane, which is detected visually. Contact is detected swiftly thereby minimizing the deflection distance of the end-effector on the substrate, allowing the end-effector to retract from the surface minimizing permanent deformation of the end-effector. 


\subsection{Materials and Methods}

\subsubsection{Nanomanipulation System}

The system consists of a nanomanipulation system (Zyvex S100) mounted onto the specimen stage of an SEM (Hitachi S-4000), as shown in Figure 3.1. There are four quadrants of 3-DOF nanomanipulators, each of which is composed of a coarse positioning stage and a fine positioning unit. The coarse positioning stage contains three identical piezoelectric slip-stick motors, having a travel range of $12 \mathrm{~mm}$ with $100 \mathrm{~nm}$ resolution. For fine positioning, the system is equipped with a piezoelectric tube having a travel range of $10 \mu \mathrm{m}$ along the axis of the tube and $100 \mu \mathrm{m}$ along each of the two orthogonal directions with $5 \mathrm{~nm}$ resolution.

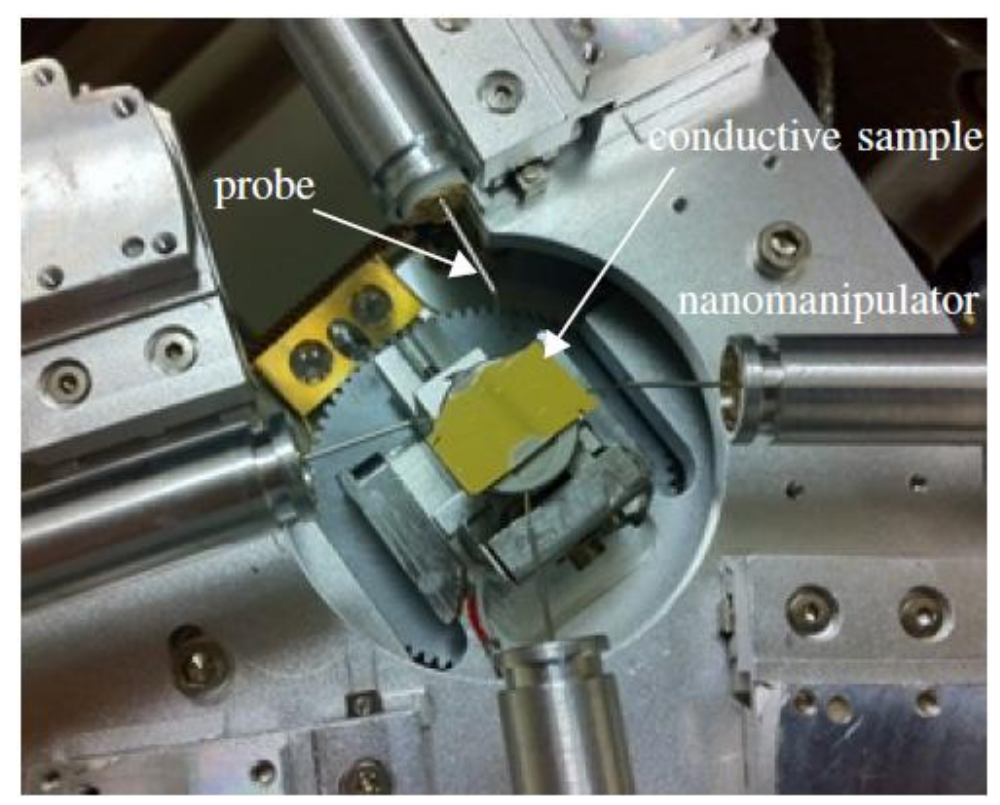

Figure 3.1: Nanomanipulators equipped with nanoprobes inside SEM.

Tungsten nanoprobes were mounted onto the nanomanipulators as end-effectors. Before loading the probes onto the nanomanipulators, the probes were chemically cleaned to remove the native tungsten oxide using $\mathrm{KOH}$ solutions and $\mathrm{HF}$. After the cleaning procedure, the probe tips had a radius of $100 \mathrm{~nm}$ and were installed at a tilting angle of $45^{\circ}$ from the substrate. Electrical 
connections were established for the nanoprobes and substrate via the feed through ports on the SEM.

\subsubsection{Visual Tracking}

The nanoprobe tip is visually tracked with a normalized cross-correlation template matching method that is robust to additive noise and to the brightness/contrast changes inherent in video-rate SEM images. The pixel intensity of an image and the template image can be represented by $I(x, y)$ and $T\left(x^{\prime}, y^{\prime}\right)$, where $(x, y)$ is the pixel position in the image coordinate frame, and $\left(x^{\prime}, y^{\prime}\right)$ is the pixel position in the template image coordinate frame, as shown in Figure 3.2.

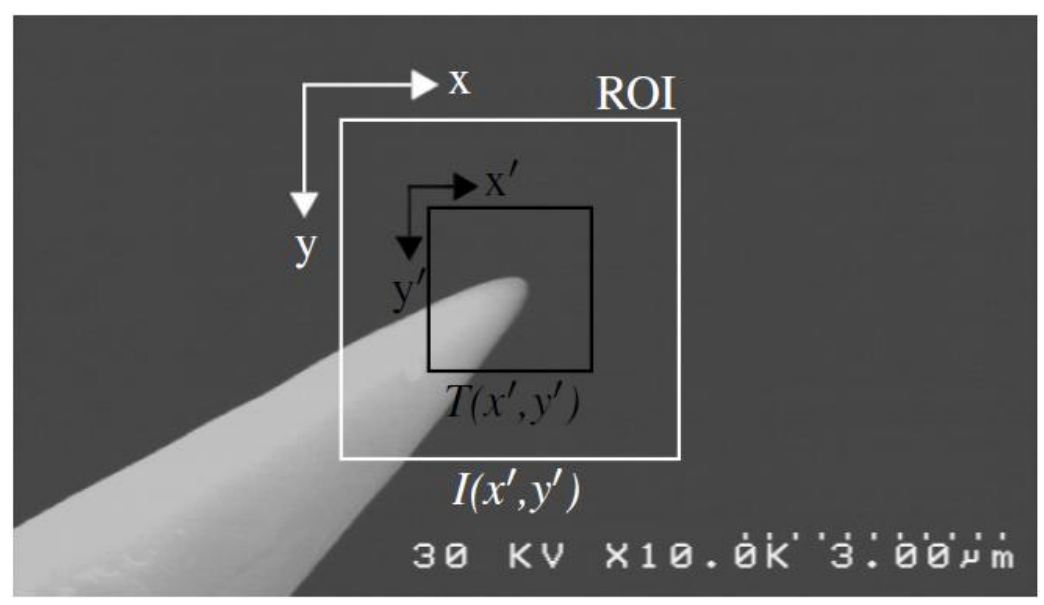

Figure 3.2: Nanoprobe tip is visually tracked using normalized cross correlation template matching. $T(x, y)$ is the template image. A search window within the proximity of the probe tip is chosen as the ROI, $I(x, y)$.

A rectangular region of the image containing the probe tip is initially recorded as the template. The template image is overlaid onto the input image at $(x, y)$, and the correlation value is computed as 


$$
R_{n c c}(x, y)=\frac{\sum_{x^{\prime}, y^{\prime}}\left[T\left(x^{\prime}, y^{\prime}\right) \cdot I\left(x+x^{\prime}, y+y^{\prime}\right)\right]}{\sqrt{\sum_{x^{\prime}, y^{\prime}} T\left(x^{\prime}, y^{\prime}\right)^{2} \cdot \sum_{x^{\prime}, y^{\prime}} I\left(x+x^{\prime}, y+y^{\prime}\right)^{2}}}
$$

where the numerator is the correlation measure, and the denominator is a normalizing factor. Within a search window of $150 \times 150$ pixels, the location with the maximum correlation value represents the new coordinate of the nanoprobe tip being tracked. The search window size is chosen by taking into account SEM charging and image drift. Eight neighboring pixels as well as the selected pixel are used to fit a curved surface to achieve sub-pixel tracking resolution [64].

In SEM imaging, image drift can be significant, particularly at high magnifications as the effects of charge drift on a specimen and electromagnetic interference from the environment become more apparent [57]. By tracking a stationary reference feature and the nanoprobe tip simultaneously, the system is able to effectively compensate for image drift and accurately determine the location of the moving nanoprobe.

\subsubsection{Vertical Depth Estimation - Depth From Focus}

The large depth of field in SEM imaging makes it difficult to accurately bring the probe into the exact co-plane as the target surface, purely based on focus information. However, high speed and simplicity of the depth-from-focus method is suitable for long-range, fast approach of the nano probe to the target surface.

Prior to contact detection, the nanomanipulators are positioned above the target surface. Since the target surface is in focus, the probe appears blurry. The probe is visually tracked as it descends towards the substrate and gradually appears sharper. Sharpness of the probe tip is quantified by calculating the normalized variance of the image, $I(x, y)$, 


$$
F_{n v}=\frac{1}{H \cdot W \cdot \mu} \sum_{\text {Height }} \sum_{\text {Width }}(I(x, y)-\mu)^{2}
$$

where $H$ and $W$ are the height and width of the ROI of the probe being tracked, and $\mu$ is the normalizing factor representing mean intensity of the image. By monitoring the normalized variance of the probe tip as the nanomanipulator descends towards the target surface, the system is able to quickly position the probe tip within the depth of field, after which the system starts the fine contact detection process.

\subsubsection{Contact Detection}

The depth-from-focus process brings the probe tip within the depth of field. The nanomanipulator then continues to lower the probe when the system performs vision-based contact detection, a method extended from optical microscopy [65]. The vision-based contact detection method is based on the experimental observation that after contact is established, further downward motion causes the end-effector to slide on the target surface, as illustrated in Figure 3.3. In this study, electrical connections to the nanoprobe and to the substrate are both accessible for electrically verifying the establishment of contact, which is discussed in detail in the next section.

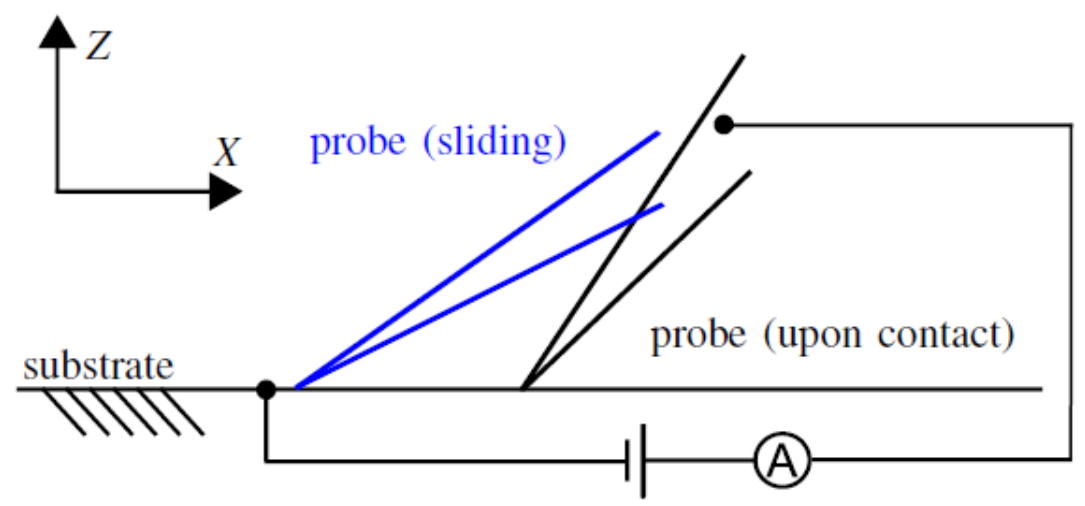


Figure 3.3: Probe slides on the substrate after contact is established, which translates to horizontal motion in the image plane. Electrical connections to the probe and substrate are for electrical verification of contact.

When the end-effector descends towards the target surface, its vertical $Z$ motion in the world coordinate translates itself into planar motion in the image plane, $x y$, according to the perspective projection model of the SEM [66]. Once contact is established between the endeffector and the target surface, an abrupt shift of motion in the image frame is recognized as the contact position.

\subsection{Experimental Results and Discussion}

\subsubsection{Depth from Focus}

When the probe descends, the system visually tracks the probe tip and monitors the normalized variance (Error! Reference source not found.). To reduce noise in normalized variance (NV), NV is averaged with past $\mathrm{N}$ frames (e.g., $N=8)$ at each image frame $i(i>N)$, according to

$$
N V_{a v g}^{i}=\frac{1}{N+1} \sum_{j=i-N} N V_{j}
$$

The averaged NV curve (Figure 3.4) is comprised of three characteristic segments, representing each phase of probe motion relative to the depth of field. In the first segment, prior to $A$, the probe approaches the depth of field and therefore, normalized variance values increase as the probe becomes sharper. In the segment between $A$ and $B$, the probe is within the depth of field. After $B$, the probe moves away from the depth of field, and the normalized variance value decreases. 
The probe in practice cannot travel the full depth of field when the target substrate is placed within the depth of field, since the probe would collide into the substrate. Hence, the system monitors the NV slopes to determine when the probe enters the depth of field and when to stop the probe from descending further by comparing the NV slopes with a threshold $k \sigma$ where $\sigma$ is the standard deviation of the NV slopes, and $k$ is a positive integer.

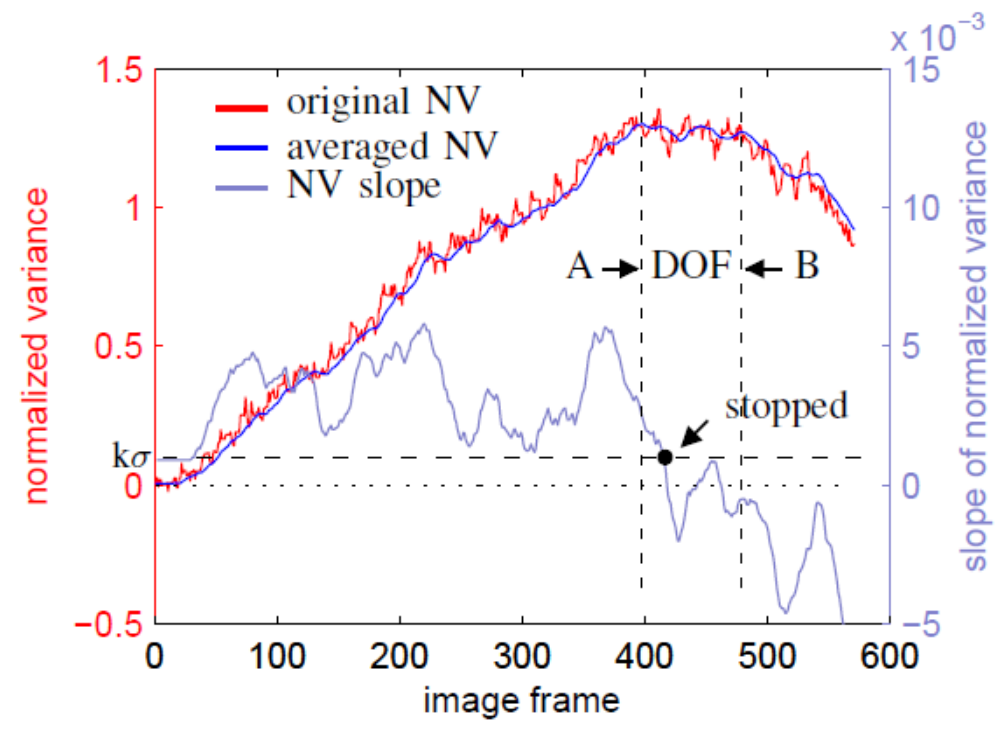

Figure 3.4: Normalized variance (NV) changes as the probe descends at $100 \mathrm{~nm}$ per image frame at 10k magnification and $5 \mathrm{kV}$ accelerating voltage. Between $A$ and $B$, the probe is within the depth of field (DOF). Slope is used to determine when the probe is within DOF. The probe stops when the normalized variance slope reaches a threshold of $k \sigma$ where $\sigma$ is the standard deviation of the slope and $k$ is a positive integer.

A higher imaging magnification is accompanied by a smaller depth of field [67]. In the system setup used in this work, at 10k magnification, the depth of field is $4.05 \mu \mathrm{m}$. At $50,000 \times$, the depth of field is $1.55 \mu \mathrm{m}$. When a large threshold coefficient $k$ is selected, the probe ends up in a position closer to $A$ in Figure 3.4, which means in practice that the probe is further above the 
target substrate surface. Hence, the risk of probe-substrate collision is low. For example, when $k=1$ was selected, the probe stopped at $1.03 \mu \mathrm{m}$ away from $A$ in Figure 3.4. When $k$ was set to be 7 , the probe stopped at $0.69 \mu \mathrm{m}$ away from $A$.

\subsubsection{Contact Detection}

Fine contact detection is initiated after the system positions the probe within the depth of field. In fine contact detection, the probe is visually tracked while it descends at a constant speed towards the substrate. The probe and the conductive substrate in experiments were both electrically connected to a source measure instrument (Keithley System SourceMeter 2602) as a calibration means for contact detection via monitoring electrical current changes.

Figure 3.5 shows a set of data captured during vision-based contact detection as well as electrical current changes before and after contact. The probe tip's pixel coordinate decreases as the probe descends towards the substrate. When the probe starts to slide abruptly forward on the substrate after contact (point $B$ in Figure 3.5), the probe tip's pixel coordinate starts to increase. At point $C$, the system determines that the probe-substrate contact has been established at $B$ since the increase in pixel coordinate has exceeded a preset threshold value. After point $C$, the pixelcoordinate decreases as the probe is raised by the nanomanipulator to the contact position, $D$. From the contact position $D$, the probe can ascend by a user-specified distance, $z_{d}$, above the substrate, and stops at $E$ to be ready for performing nanomanipulation tasks. 


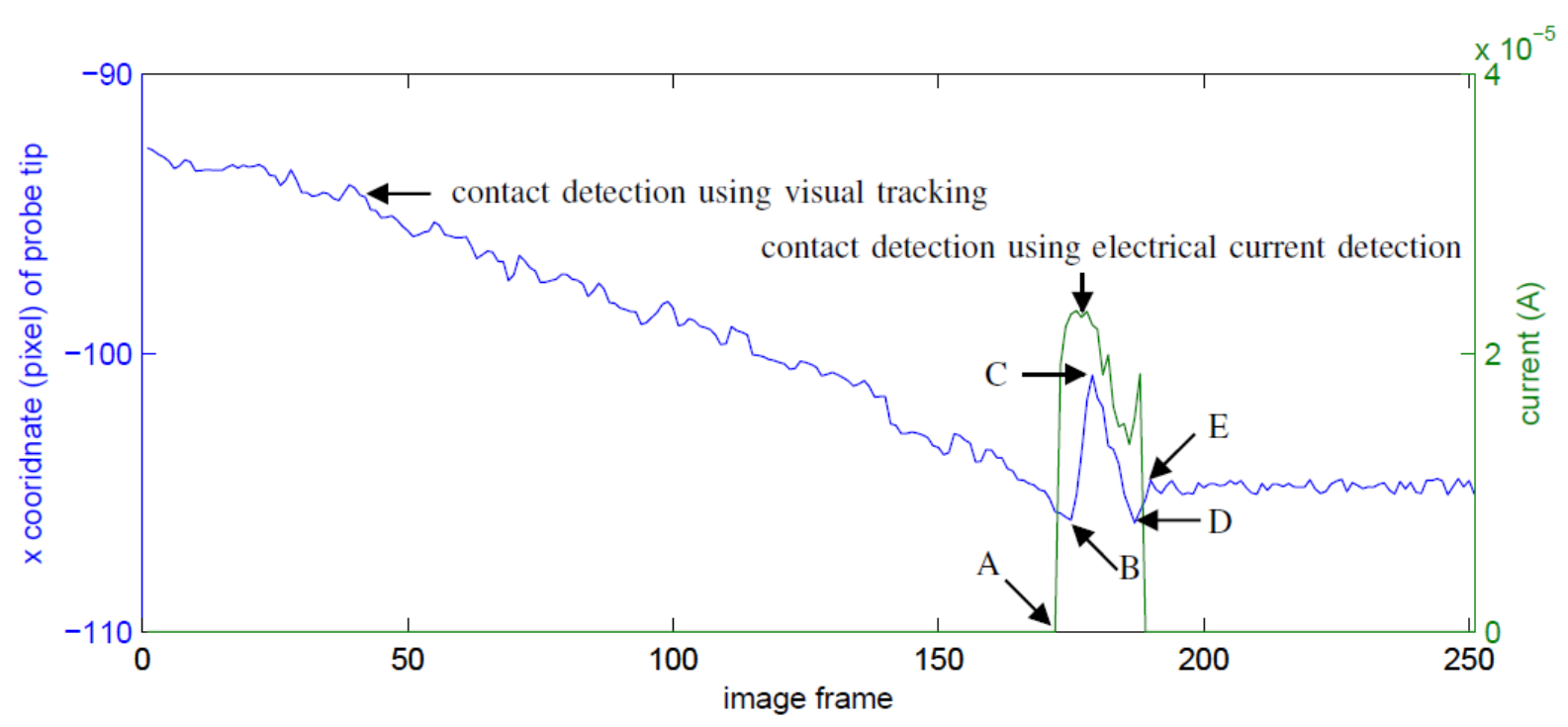

Figure 3.5: Contact detection and subsequent $Z$ positioning of a probe via sub-pixel normalized cross correlation tracking (SEM: $10 \mathrm{kV}$ accelerating voltage, $10 \mathrm{k}$ magnification; motion step size produced by nanomanipulator: $10 \mathrm{~nm}$ ). Electrical current measurement is used to verify contact between the probe tip and conductive substrate.

During the contact detection process, electrical current measurements were also made. As shown in Figure 3.5, contact was detected electrically at $A$ before it was visually recognized at $B$ after three additional $10 \mathrm{~nm}$ steps. This delay is from the probe experiencing deformation before friction between the probe and the substrate was overcome. The probe slides forward and continued to descend until $C$ when the probe was raised by the nanomanipulator to the contact position $D$. Further upward motion of the nanomanipulator lifted the probe off the substrate, and electrical current disappeared.

The electrical current measurement effectively verified visual contact detection results. Tunneling current between the probe tip and conductive substrate at $A$ in Figure 3.5 prior to visual contact detection at $B$ was not likely. Firstly, the probe descent step size used in fine contact detection $(10 \mathrm{~nm})$ is substantially larger than the tip-to-sample distance at the level of 
angstroms required to induce tunneling current, as used in scanning tunneling microscopy (STM). Secondly, STMs generally operate with a $10 \mathrm{mV}$ to $1 \mathrm{~V}$ bias [68] compared to the $1 \mathrm{mV}$ used in our experiments. Finally, the tunneling current induced in STMs is generally in the range of $0.2 \mathrm{nA}$ to $10 \mathrm{nA}$, which is substantially smaller than the $\mu \mathrm{A}$ level shown in Figure 3.5.

In consideration of nanomanipulation tasks where the end-effector must interact with an object, the accuracy of the vision-based contact detection algorithm is defined as the difference between the desired distance of the probe from the substrate, $z_{d}$, and the actual distance of the probe from the substrate, $z_{a}$. To determine the actual distance of the probe from the substrate after contact detection, gold nanospheres of $\sim 100 \mathrm{~nm}$ in diameter were deposited onto a conductive gold substrate. Once the system completed the contact detection process, the probe was positioned at a distance, $z_{a}$, from the substrate near a gold nanosphere with a diameter, $D$, as shown in Figure 3.6(a). The probe approached the nanosphere laterally in the $X Y$ plane while probe-nanosphere contact was detected by monitoring the electrical current between the probe tip and substrate, as shown in Figure 3.6(b). To minimize the effect of height variations across the substrate, the probe was positioned in direct proximity of the nanosphere to reduce the lateral distance traveled by the probe to the nanosphere. 


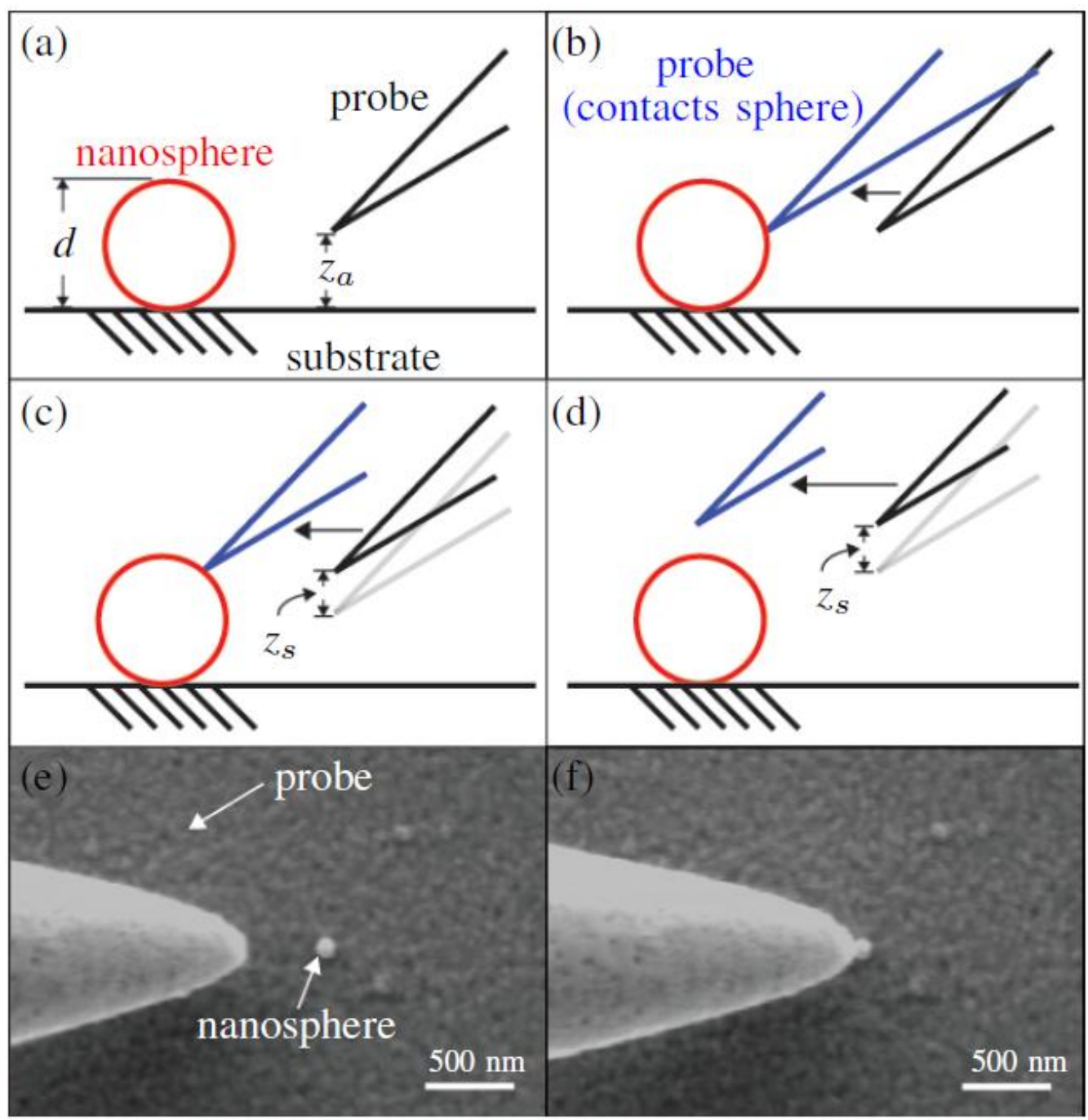

Figure 3.6: Quantification of contact detection accuracy. (a) Probe is initially positioned above the substrate at a distance, $z_{a}$, after vision based contact detection is completed. (b) Probe moves laterally towards nanosphere to establish contact which is verified via electrical current monitoring. (c) Probe is raised by one step, $Z_{s}$ and laterally moved again to touch the nanosphere. (d) This process is repeated until the probe is raised high enough, and no longer comes in contact with the nanosphere. (e)(f) SEM images captured in the probe nanosphere contact experiments. Nanosphere was $100 \mathrm{~nm}$ in diameter. 
When the probe passes over the nanosphere without touching the nanosphere, no current is detected, and the probe is known to be positioned at a vertical distance greater than the diameter of the nanosphere. If a current is detected, the probe must have a vertical position within $D$ from the substrate. In our experiments, the probe was raised by $10 \mathrm{~nm} / \mathrm{step}$ from position $D$ in Figure 3.5 to repeatedly contact the nanosphere, as illustrated in Figure 3.6(c). This process was repeated until the $k^{\text {th }}$ incremental step when the probe passed over the nanosphere, and probe-nanosphere contact (electrical current) was lost (Figure 3.6(d)). Accuracy, therefore, is $\left\|d-k * Z_{s}-z_{d}\right\|$, where $z_{s}=10 \mathrm{~nm}$ is the step increment as shown Figure 3.6(c)(d), and $z_{d}$ is the desired distance of the probe from the substrate. Figure 3.6(e)(f) show the experimental situation of probe-nanosphere contact.

Besides accuracy, the maximum distance the probe tip is deflected during contact detection (i.e., between $A$ and $C$ in Figure 3.5) was also quantified in experiments and is defined as the deflection distance. Table 3.1 and Table 3.2 summarize testing results of the deflection distance and contact detection accuracy at different magnifications and accelerating voltages. Each data point in the tables was from 50 trials under each condition. Table 3.1 shows that higher magnifications resulted in higher contact detection accuracy, since each pixel represents a smaller physical dimension allowing more accurate contact detection from SEM visual feedback. Contact detection accuracy was not significantly affected by accelerating voltages (see Table 3.2), which demonstrates that normalized cross correlation is a robust tracking method against changes in surface textures associated with varying accelerating voltages [69]. 
Table 3.1: Contact detection accuracy and deflection distance at varying magnifications. Accelerating voltage: $10 \mathrm{kV}$.

\begin{tabular}{cccccc}
\hline \hline magnification & $3 \mathrm{k}$ & $5 \mathrm{k}$ & $10 \mathrm{k}$ & $20 \mathrm{k}$ & $50 \mathrm{k}$ \\
\hline accuracy $(\mathrm{nm})$ & 63.98 & 49.77 & 43.36 & 33.84 & 21.53 \\
deflection distance $(\mathrm{nm})$ & 223.07 & 157.88 & 93.61 & 63.28 & 26.76 \\
\hline
\end{tabular}

Table 3.2: Contact detection accuracy and deflection distance at varying accelerating voltages. Magnification: $20 k$.

\begin{tabular}{ccccc}
\hline \hline accelerating voltage & $1 \mathrm{kV}$ & $10 \mathrm{kV}$ & $20 \mathrm{kV}$ & $30 \mathrm{kV}$ \\
\hline accuracy $(\mathrm{nm})$ & 31.39 & 33.84 & 32.81 & 30.16 \\
deflection distance $(\mathrm{nm})$ & 64.69 & 63.28 & 66.39 & 59.76 \\
\hline
\end{tabular}

Table 3.2 also shows that the deflection distance of the probe was not affected by changes in accelerating voltages. However, the deflection distance was heavily dependent on the magnification used in contact detection (see Table 3.1). Deflection distance decreases as SEM magnification increases, again because of a smaller physical dimensional each pixel represents and the contact detection approach relies on the detection of image pixel changes. Probe damage due to deflection distances will be discussed in Section $C$.

In this work, repeatability of the contact detection method is defined as the frequency at which better than $100 \mathrm{~nm}$ accuracy is achieved. From 500 trials of vision-based contact detection, repeatability was determined to be $98.8 \%$. The $1.2 \%$ failure cases all occurred under the $3 \mathrm{k}$ magnification. The limited imaging resolution at low magnifications made the algorithm unable to converge. Thus, a magnification higher than $5 \mathrm{k}$ should be used for the vision-based contact detection method to achieve both high repeatability and high accuracy. 


\subsubsection{End-Effector Damage}

End-effector damage is difficult to avoid in manual operation of nanomanipulators using joysticks. Figure 3.7 shows end-effector damage after manually performing a nanomanipulation task. Probe damage is less of an issue when the system conducts automated contact detection due to sub-pixel image processing capability and faster response after contact is established. Experiments were performed to quantify probe tip deformations after $n=200$ trials of visionbased contact detection at 20k magnification. After 200 times contact detection trials, the side profile of the probe tip was registered with the contour of the probe tip captured before contact detection trials (see Figure 3.8). The probe tip experienced permanent deformation of $22.6 \mathrm{~nm}$ after 200 contact detection trials. Although the deflection distance of the probe was $63.28 \mathrm{~nm}$ at 20k magnification (Table 3.1), permanent deformation was much smaller (22.6 $\mathrm{nm}$ ) due to the elasticity of the probes.

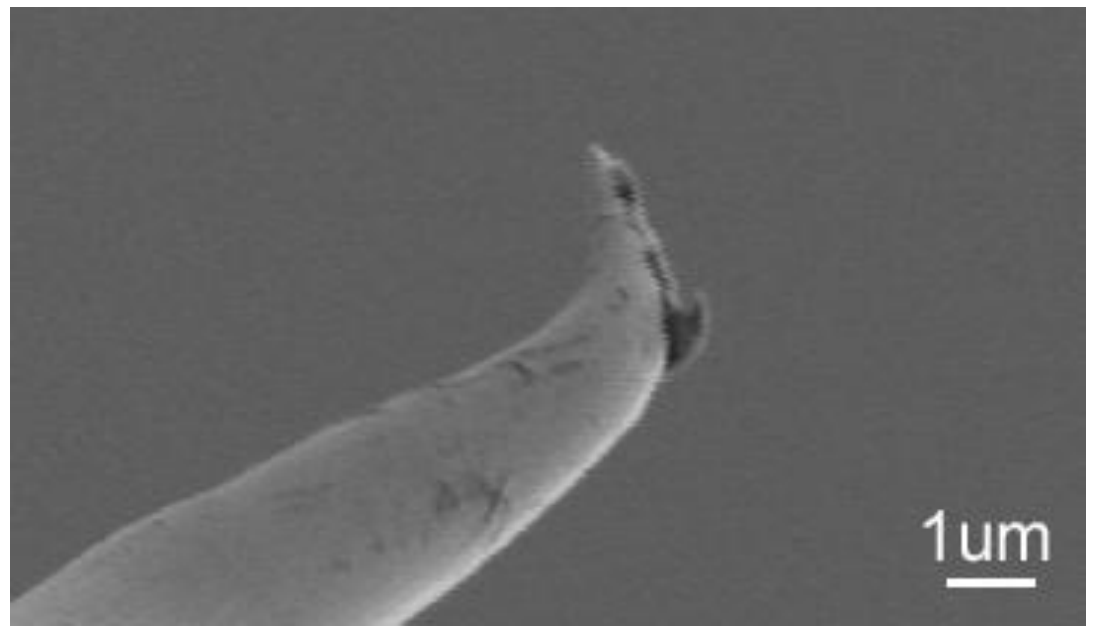

Figure 3.7: Damaged nanoprobe resulting from manual manipulation. 


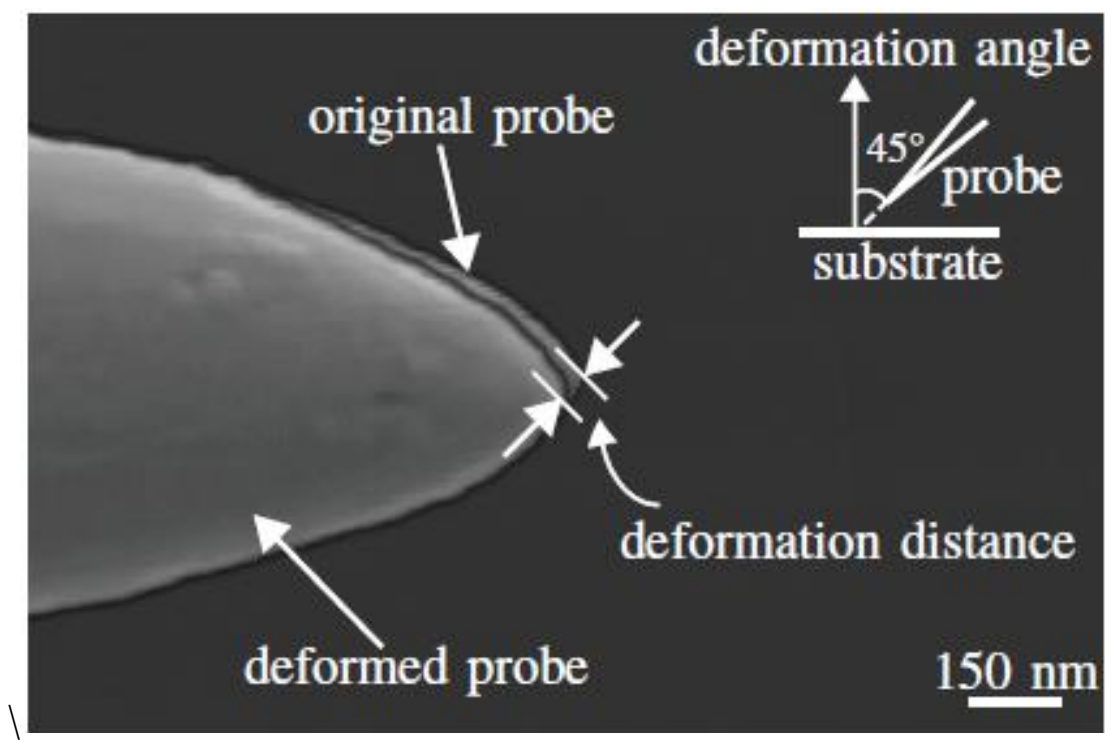

Figure 3.8: Measurement of permanent deformation of the probe tip before and after 200 times contact detection.

\subsubsection{Conclusion}

This chapter described a method for detecting contact between an end-effector and a target surface using SEM as a vision sensor. Contact detection is a critical issue in manipulating nano-scaled objects inside SEM. Different from previous methods that replied on additional touch/force sensors or specialized hardware modifications to a standard SEM, this SEM visionbased contact detection approach is compatible for operation with all standard SEM, is able to achieve high repeatability and accuracy, and automatically completes contact detection (depthfrom-focus and fine contact detection combined) within typically 60 seconds. This study systematically evaluated the performance of the contact detection approach under various imaging conditions. Permanent deformation of probe tips was also quantified. 


\section{Chapter 4 \\ Contact Detection in Nano Device Construction through Automated Nanomanipulation}

\subsection{Introduction}

Nanowire field-effect transistors (nano-FETs) are emerging as ultrasensitive, miniaturized, label-free sensors in detecting exceedingly low concentrations of chemical and biological molecules for medical and environmental applications (e.g., proteins [7] , nucleic acids, [8, 9], viruses[10], $\mathrm{O}_{2}[3], \mathrm{NO}_{2}$ [4], $\mathrm{NH}_{2}$ and humidity [5]). Top-down micro- and nanofabrication is often used for silicon nanowire sensor construction. These fabrication methods include electron-beam nanolithography followed by dry etching [7], silicon wire thinning via repeated surface oxidation and $\mathrm{HF}$ etching processes $[12,13]$ and anisotropic timed etching of silicon structures [14]. These top-down techniques involve high processing costs/complexity and low yields associated with e-beam lithography and focused ion beam (FIB), and significant variability across etched devices due to etching non-uniformity across the wafer and high sensitivity to processing conditions.

Alternatively, pre-synthesized nanostructures (i.e. nanotubes and nanowires) are of low cost and can be integrated into micro-structures to form nano-FET sensors. Existing wafer-scale nanowire/nanowire integration methods, such as directed assembly [15], contact printing [16], and dielectrophoresis [17] are inexpensive; however, they are incapable of precisely controlling nanostructure parameters, such as nanostructure number and diameter, as shown in Figure 4.1. These nanostructure attributes have been shown in nanowire electrical transport studies [18, 19], gas-phase chemical sensing [20, 21], aqueous sensing of $\mathrm{pH}$ and ionic species [22, 23], and 
nanoribbon FETs $[24,25]$ to influence device performance. Therefore these nanowire/nanotube integration methods will result in devices with higher device-to-device variability.

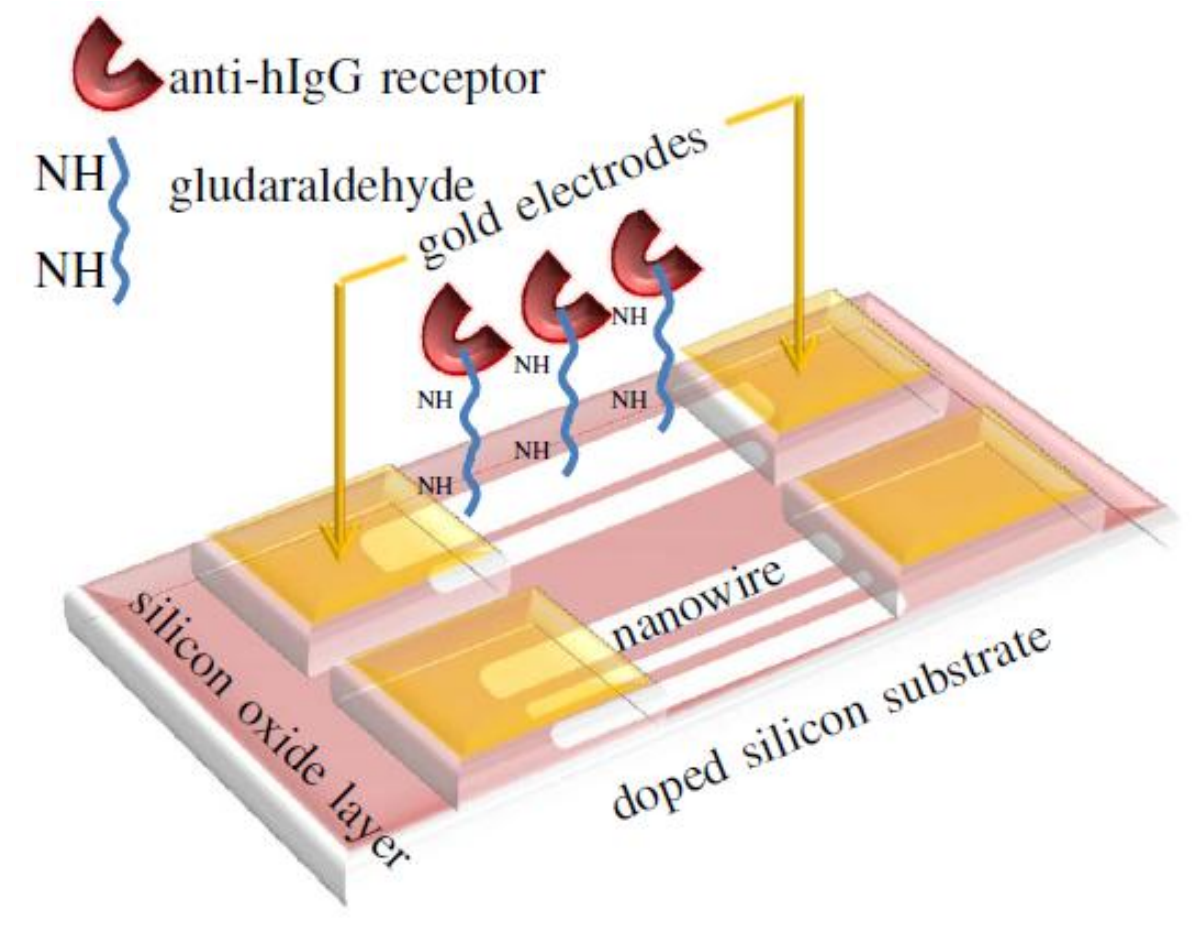

Figure 4.1: Schematic of nano-FET sensors for sensing hIgG protein. Nanowire diameter and number vary across devices, resulting in sensitivity variations.

By contrast, mechanical nanomanipulation, despite being serial in nature and slower compared to the abovementioned nanostructure integration methods, promises specificity, precision, and programmed motion. In addition, using a vision sensor allows us to selectively manipulate nanostructures based on their characteristics (i.e., number, diameter, etc.). A combined use of wafer-scale processes and automated nanomanipulation would constitute a new paradigm to bring nanomanufacturing one step further towards scaled-up operation and in the meanwhile, achieve high precision control of nanostructure parameters. 
This paper presents a technique for nano-FET device construction based around the contact detection algorithm discussed in the previous chapter. We use wafer-scale processes for throughput followed by automated nanomanipulation to perform post processing for precisely controlling nanowire number and diameter. Visual recognition and tracking using SEM as a vision sensor as well as vision-based closed-loop position control enable the system to post process nano-FET devices at a speed of $\sim 1 \mathrm{~min} /$ device with a success rate of $95 \%(\mathrm{n}=500)$. The device is encapsulated by a microfluidic chamber for protein sensing. Using this technique, we are able to fabricate arrays of nano-FET biosensors with carefully controlled nanowire properties for subsequent investigation of their effects on device performance (i.e., sensitivity) variability. Although theoretical framework exists in qualitatively understanding the influence of nanowire diameter and number $[70,71]$ on device sensitivity, they have not yet been experimentally quantified.

\subsection{System and Device Batch Microfabrication}

\subsubsection{Nanomanipulation System}

The nanomanipulation system (Figure 4.2(a)) contains two independent 3-DOF nanomanipulators, both assembled from three linear coarse-fine nanopositioners equipped with optical encoders (resolution: $2 \mathrm{~nm}$ ). The nanopositioners are capable of operating in either the stick-slip (coarse) mode to produce a large motion range $(10 \mathrm{~mm})$ with a step size of $\sim 100 \mathrm{~nm}$, or in the fine mode with a $1 \mu \mathrm{m}$ travel range and a resolution of $1 \mathrm{~nm}$ [72]. 


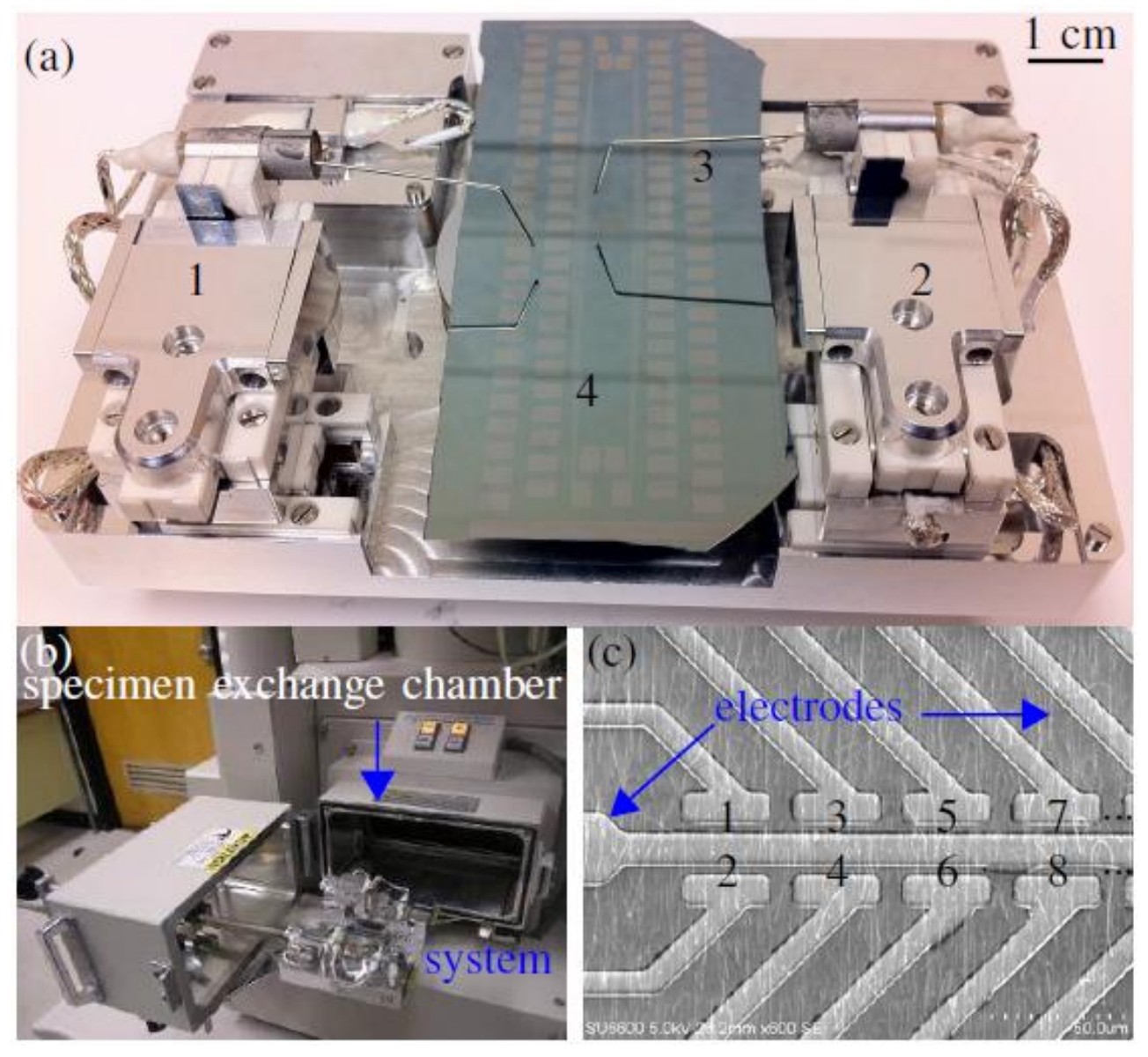

Figure 4.2: (a) Nanomanipulation system. 1. nanomanipulators-1, 2. nanomanipulators-2, 3. nanoprobe, 4. Batch fabricated nano-FET array mounted on the SEM stage for nanomanipulation. (b) The system is mounted onto and demounted from the SEM through the specimen exchange chamber. (c) SEM image of 8 nano-FET devices where microelectrodes are on top of the nanowires and pin the nanowires to the substrate.

The system is small in size $\left(100 \times 80 \times 46 \mathrm{~mm}^{3}\right)$ and capable of being mounted onto and demounted from a standard SEM (e.g., Hitachi SU6600) through the specimen exchange chamber (Figure 4.2 (b)) without breaking the high vacuum inside the SEM. The nanomanipulators can be operated manually via joysticks or via computer control. Tungsten nanoprobes (tip diameter: $200 \mathrm{~nm}$ ) were chemically cleaned to remove the native tungsten oxide 
using $\mathrm{KOH}$ solutions and $\mathrm{HF}$ before they are mounted onto the nanomanipulators. Electrical connections from the nanomanipulators to the outside of the SEM were established through a feed-through port on the SEM.

\subsubsection{Device Batch Microfabrication}

Phosphorous-doped (n-type) silicon nanowires (doping concentration of $10^{17}$ and $10^{19}$ atoms $/ \mathrm{cm}^{3}, 40-130 \mathrm{~nm}$ in diameter) were synthesized using a chemical vapor deposition. Asgrown nanowires were deposited and aligned along a single direction onto a silicon substrate with a $200 \mathrm{~nm}$-thick oxide layer via nanowire contact printing [16]. The growth substrate placed on top of the device substrate such that nanowires were sandwiched in between the two silicon wafers. A weight was then placed above the growth substrate to provide a normal force of 20

$\mathrm{g} \cdot \mathrm{cm}^{-2}$. The growth substrate slides over the device substrate at a constant velocity of 20 $\mathrm{mm} \cdot \mathrm{min}^{-1}$ thereby transferring of a high-density monolayer of well-aligned nanowires on to the device substrate. The amount of nanowires transferred depends on the pressing force between the growth and receiving substrates, nanowire density on the growth substrate, and the surface properties of the receiving substrate.

Aluminum electrodes, $200 \mathrm{~nm}$ thick, are formed on top of the nanowires using standard lithography, metallization, and liftoff microfabrication processes to produce arrays of multinanowire transistors. Figure 4.2 (c) shows eight electrode pairs with bridging nanowires. Each transistor consists of numerous nanowires bridging the source-drain electrode pair. Nanowires are orientated parallel to one another along the printing direction resulting in an effective length of $\sim 5 \mu \mathrm{m}$ as dictated by the electrode pair gap. The thick Al electrodes act to firmly pin the nanowires to the substrate to facilitate easy nanowire removal. 


\subsection{Manual Nanomanipulation}

\subsubsection{Procedures}

Manual nanomanipulation for post processing the wafer-scale fabricated nano-FET devices consists of four major steps as depicted in Figure 4.3. An array of nanowire FET devices (sensors) is first mounted onto the nanomanipulation system and loaded into the SEM. Each device consists of an electrode pair with numerous bridging nanowires that are pinned firmly in place underneath the electrodes, as shown in Figure 4.2 (c). After examination of the nanowire diameters present on each device, the operator selects a suitable nanowire to keep ('target nanowire'). Preference is given to those nanowires that are located farthest from its neighbors to minimize the likelihood of contact with other nanowires or nanoprobes during the nanowire removal process. The nanowires to be removed are termed 'undesired nanowires'.
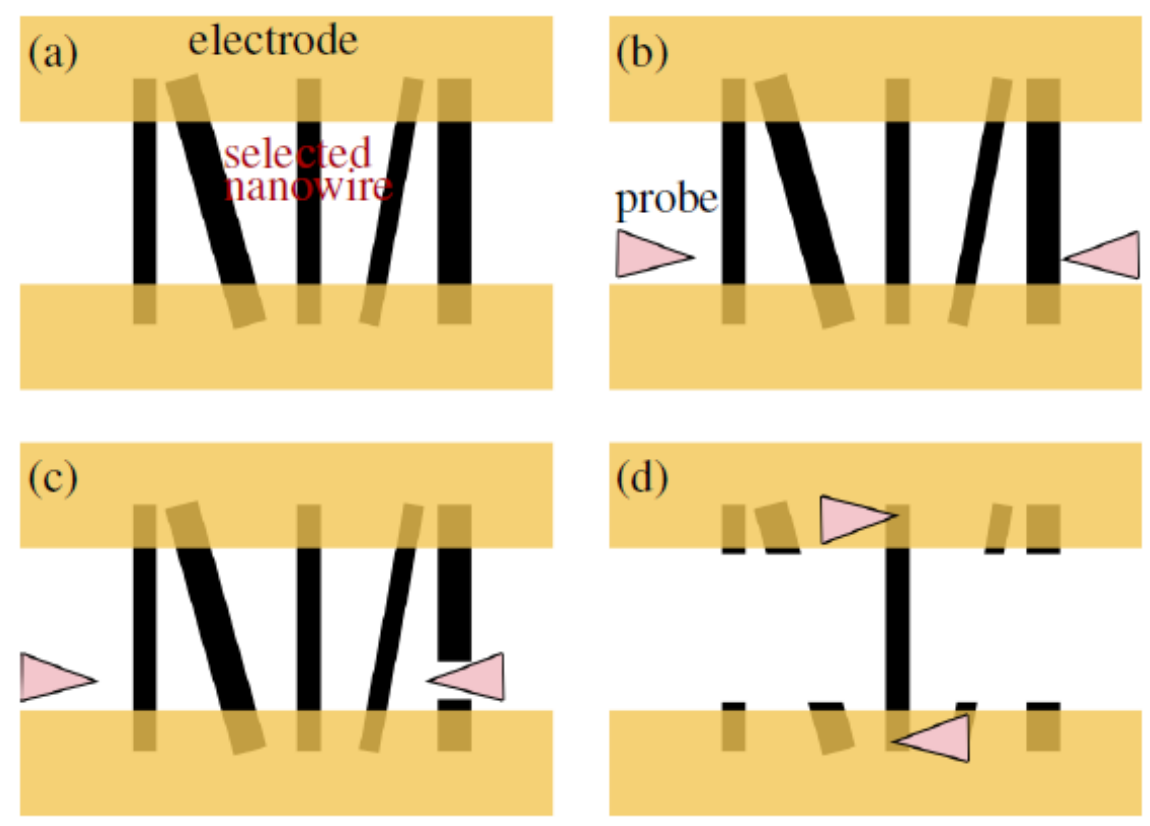

Figure 4.3: Physical removal of selected nanowires. (a) Operator decides which nanowire(s) to keep. (b) Lower nanoprobes to the substrate surface. (c) Sever undesired nanowires. (d) Perform electrical characterization. 
Before physical removal of undesired nanowires using the nanoprobes mounted on the nanomanipulators, the operator lowers the nanoprobes until the operator judges that nanoprobes have established contact with the device substrate. As illustrated in Figure 4.3 (c), the operator then uses the joystick to position the nanoprobe tip along the two inner edges of the electrodes for severing undesired nanowires. Since the nanowires are firmly pinned underneath the electrodes, nanowire fracture during selective removal is assured to occur along the electrode edges.

Physical interactions with the nanoprobe cause nanowires to bend prior to fracture. A bent nanowire may come into contact with adjacent nanowires and, in some cases, cause physical damage to the target nanowire. A severed nanowire may land on top of the target nanowire, forming an undesired nanowire network. Significant care must be taken by the operator when severing adjacent nanowires in close proximity to each other in order to (1) reduce inadvertent damage to the target nanowire due to imprecise nanoprobe movements associated with manual operation control, and (2) reduce the possibility of nanowire networking.

Subsequent to nanowire removal (Figure 4.3 (d)), the operator can conduct in situ electrical characterization of the target nanowire by placing the two nanoprobes onto the source and drain electrodes. The operator can then confirm Ohmic contact and measure the target nanowire's $I$ - $V$ properties. Devices with significant contact resistance are deemed inappropriate for sensing use and are thus discarded.

\subsubsection{Success Rate and Efficiency}

Success rates and the average time spent processing each device are operator dependent in manual nanomanipulation. Table 4.1 summarizes the success rate and average processing time 
for three operators. The three operators all had experience in conducting manual nanomanipulation, and each processed 20 devices to form single-nanowire devices. The data table also details the frequency of occurrence for the two failure cases (i.e., nanowire broken and networked nanowires).

Table 4.1: Manual nanomanipulation for post processing of 60 devices

\begin{tabular}{ccccc}
\hline \hline \multirow{2}{*}{ operator } & successful & \multicolumn{2}{c}{ unsuccessful } & average processing \\
& & broken & networked & time (min) \\
\hline 1 & 8 & 10 & 2 & 9 \\
2 & 12 & 7 & 1 & 12 \\
3 & 9 & 10 & 1 & 10 \\
\hline
\end{tabular}

The three trained operators produced success rates ranging from $\sim 40 \%$ to $\sim 60 \%$. The predominant failure case is attributed to accidental nanowire breakage, which resulted from imprecise position control of the nanoprobes. Manual nanomanipulation is also time-consuming. Averagely, it took 10.3 min for an operator to process one nanowire FET device. The total time cost of $10.3 \mathrm{~min}$ is from $\sim 4.5 \mathrm{~min}$ for nanowire selection, $\sim 3.2 \mathrm{~min}$ for contact detection, and $\sim 2.6$ min for nanowire removal.

\subsection{Automated Nanowire Removal}

To improve nanomanipulation success rate and efficiency, visual recognition of nanowires and SEM vision-based position control were developed to automate nanowire FET device post processing, including nanowire detection and selection, contact detection, and nanowire severing.

\subsubsection{Automated Post Processing}

As illustrated in Figure 4.4(a), a multi-nanowire device has $n$ bridging nanowires. The parameters associated with each nanowire $N W_{i}$ include the nanowire diameter $\left(d_{i}\right)$, and the 
distance to the adjacent nanowires ( $\Delta s_{\text {left }}^{i}$ and $\Delta s_{\text {right }}^{i}$ for the adjacent distances to the left and right, respectively). A nanowire whose $\Delta s_{\text {left }}^{i}$ and $\Delta s_{\text {right }}^{i}$ values are larger than a minimum threshold $\Delta s_{\min }$ can be considered a suitable target nanowire candidate. $\Delta s_{\min }$ is the minimum separation distance between two nanowires, which is determined experimentally. Defining $\Delta s_{\min }$ is important such that removal of one nanowire would not damage to the other. Among all target nanowire candidates satisfying the $\Delta s_{\text {min }}$ requirement, the nanowire whose diameter is the closest to the desired diameter $\left(d_{r}\right)$ is selected by the system as the target nanowire (i.e., nanowire to keep). In summary, automated nanowire selection is performed according to

$$
\begin{array}{cc}
\min _{d_{i}}: & \left\|d_{i}-d_{r}\right\| \\
\text { subject to }: & \Delta s_{\text {left }}^{i} \geq \Delta s_{\text {min }} \\
& \Delta s_{\text {right }}^{i} \geq \Delta s_{\text {min }} \\
& i=1, \cdots, n
\end{array}
$$




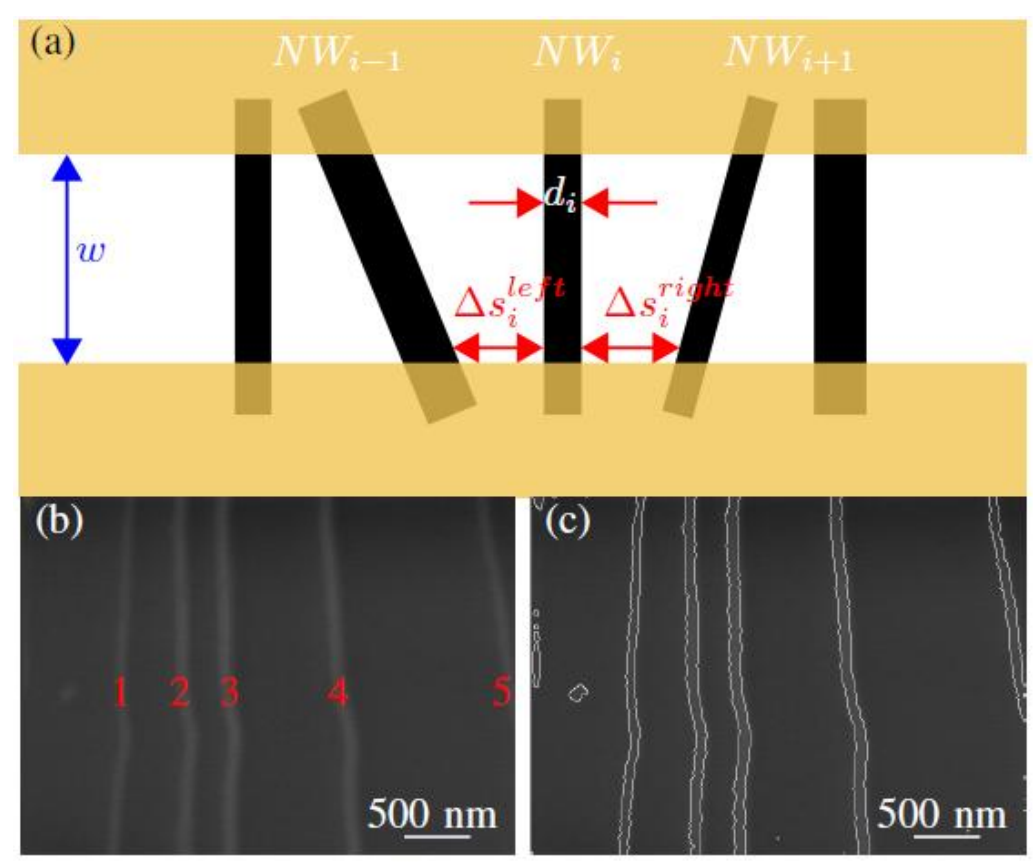

Figure 4.4: (a) Diameter and distances to adjacent nanowires are determined for each nanowire. (b) A device image showing electrode bridging nanowires (electrodes not shown in image). (c) Nanowire contours are extracted from image processing.

The system obtains contours of nanowires (see Figure 4.4(b) and (c)) using the greedy snake algorithm [73]. Nanowire networks pose a problem in device performance as their affect on device sensitivity differs than that of single bridging nanowires [21] and are identified by comparing contour widths where too wide a width, in comparison to expected nanowire diameters, indicates a nanowire with a branching nanowire attached to it. Nanowire networks are excluded from the ranking process in Equation 4.

Nanowire diameter $d_{i}$ is obtained by finding the average width of the contour of nanowire $N W_{i}$. Separation distance $\Delta s_{\text {left }}^{i}$ between nanowire $N W_{i}$ and $N W_{i-1}$ is determined by locating the shortest distance between points on the left edge of the contour of nanowire $N W_{i}$ and points on the right edge of the contour of nanowire $N W_{i-1}$ along the electrode edge direction. The system 
applies the same approach to determine $\Delta s_{\text {right }}^{i}$. Coordinates of the two end points on the contour of each undesired nanowire near the two electrode edges are also extracted for subsequent position control and nanowire removal.

\subsubsection{Contact Detection}

We summarized our automated contact detection approach in the previous chapter. Briefly, the nanomanipulation system moves a nanoprobe downwards at a constant speed. The position of the nanoprobe tip in the image frame, along its moving direction, is automatically tracked from the SEM images. After nanoprobe-substrate contact is established, further downward motion of the nanoprobe induces a sharp change of probe movement in the image coordinate frame resulting from the sliding motion of the nanoprobe on the substrate. The system detects this image coordinate change for determining the contact point between the nanoprobe and the substrate surface and positions the probe at this point. In experiments, contact detection on the device substrate is performed with the nanoprobe $\sim 3 \mu \mathrm{m}$ away from the surface.

\subsubsection{Nanowire Removal}

The system uses a look-then-move method for $X Y$ position control of the nanomanipulators. The system obtains coordinate difference between the probe and the target position in the image frame as $(\Delta x, \Delta y)$ using image processing. Through coordinate transformation, $(\Delta x, \Delta y)$ is converted to $\left(X_{d}, Y_{d}\right)$ in the world frame of the nanomanipulator. For the $X$-axis control, $X_{d}$ is the reference input to the closed-loop control system, as shown in Figure 4.5 that also applies to the $Y$ direction. Encoders integrated in the nanomanipulators provide position feedback $X_{c}$ along the $X$ direction. Due to the limited travel range of the piezo actuator, the piezo motor (coarse) is used to bring the probe to within the fine travel range to the target 
position. The piezo actuator (fine) then is switched on to bring the probe precisely to the target position. Hence, voltage input to the nanomanipulators is

$$
\begin{cases}U_{c}, & E \geq \text { travel range of piezo actuator } \\ U_{f}, & E \leq \text { travel range of piezo actuator }\end{cases}
$$

$U_{c}$ and $U_{f}$ are both generated by the PID control law.

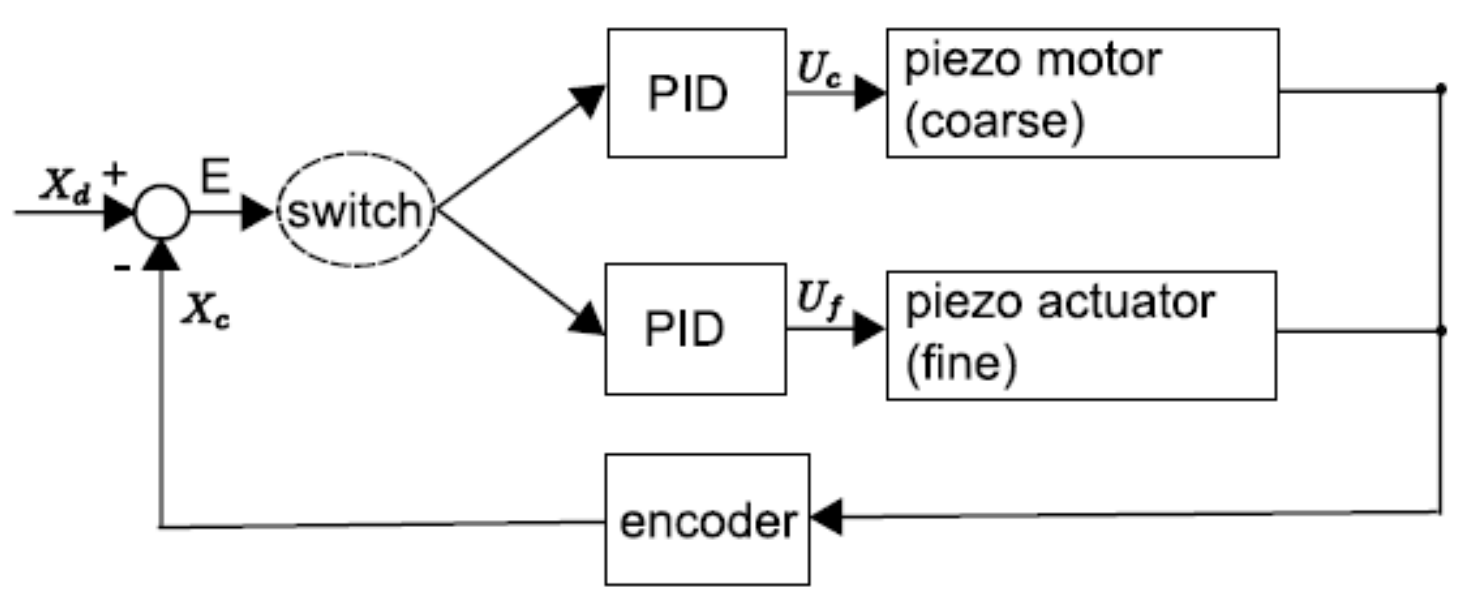

Figure 4.5: Look-then-move and closed-loop position control.

Automated nanowire removal is realized using two-probes for post-processing of fabricated biosensor devices. For a single-nanowire device, the nanoprobe establishes contact with the substrate on the bottom electrode edge of the device. Nanowires are severed inwards until the probe is positioned $\Delta s_{\min }$ away from the desired nanowire at which the probe is then pulled back to the contact point. The probe is then raised and is positioned at the upper electrode where contact detection is performed again. Nanowires are then removed on the upper electrode. The mirror of this process is repeated for the other nanoprobe to remove nanowires on the 
opposite side. The two probes then perform contact detection on the bottom and top electrodes separately for electrical testing.

\subsubsection{Incorporation of Sensors into Microfluidic Device}

Nano-FET arrays were encapsulated into a simple microfluidic channel to facilitate solution handling during surface functionalization and subsequent experimentation. Surface functionalization was performed using 2\% (3-Aminopropyl)triethoxysilane (APTES) in ethanol (30 min.), 3\% glutaraldehyde in phosphate buffered saline (0.01M PBS, pH 7.4, 30 min) and anti-human IgG (Invitrogen, USA) in PBS.[74] Unreacted terminal aldehyde groups were passivated with a 100mM ethanolamine solution in PBS (pH 8.4) and washed with a solution of 0.5\% tween-20 in PBS. Successful and repeatable functionalization was verified using x-ray photon spectroscopy (XPS) and fluorescence microscopy analysis.

\subsubsection{Protein Sensing Experiments}

All biosensing experiments were carried out at room temperature using various concentrations of hIgG in $0.1 \mathrm{X}$ PBS as a model analyte for immuno-detection applications. Increasing concentrations of $\mathrm{hIgG}$ (from $10 \mathrm{fg} \cdot \mathrm{ml}^{-1}$ up to $10 \mu \mathrm{g} \cdot \mathrm{ml}^{-1}$ ) were introduced to the nano-FET sensor and sufficient time was given to allow the signal to reach equilibrium before adding in subsequent solutions. Electrical measurements were recorded using a Keithley 2602 Sourcemeter $\left(\mathrm{V}_{\mathrm{sd}}=10 \mathrm{mV}, \mathrm{V}_{\mathrm{g}}=-1.0 \mathrm{~V}, \mathrm{I}_{\mathrm{sd}}\right)$. The gate voltage was applied using an immersed $\mathrm{Ag} / \mathrm{AgCl}$ solution gate electrode within the electrolyte.

Devices were characterized simultaneously throughout experimentation using a LabVIEW-controlled data acquisition system and a multiplexer (National Instruments, USA). For each concentration of $\mathrm{hIgG}$, the normalized device current $\left(\mathrm{I} / \mathrm{I}_{0}\right)$ was measured and plotted 
against the protein concentration. Sensitivity, defined as $\left(\mathrm{I}-\mathrm{I}_{0}\right) / \mathrm{I}_{0}$, was then determined and plotted against the test parameter. Statistical significance was determined using unpaired student t-test assuming unequal variances $(\mathrm{p}<0.05)$.

\subsection{Results and Discussion}

\subsubsection{Nanowire Selection}

The desired nanowire diameter, $d_{r}$, can be user specified for a group of nano-FET devices such that sensitivity variations across these devices are suppressed. The selection of $d_{r}$ in practice can be based on analyzing diameter distributions of nanowires throughout a growth substrate. In this study, we constructed several groups of nano-FET devices, each group having a different $d_{r}$ value. This makes sensitivity variations low within the group and much higher across groups, which is further discussed in Chapter 4.5.4.

Table 4.2: Effect of $\Delta s_{\min }$ value on number of qualified $N W s$ within each device.

\begin{tabular}{cccccc}
\hline \hline$\Delta s_{\min }(\mu \mathrm{m})$ & 5 & 3 & 2 & 1 & 0.5 \\
\hline average number of NW candidates & 1.2 & 2.5 & 4 & 7 & 12 \\
\hline
\end{tabular}

The selection of $\Delta s_{\text {min }}$ value influences success rates and also determines the number of qualified target nanowire candidates. A large $\Delta s_{\text {min }}$ value results in higher nanomanipulation success rates but a smaller number of qualified target nanowire candidates from which a nanowire with the desired $d_{r}$ can be selected. Table 4.2 summarizes the average number of qualified target nanowire candidates with each nano-FET device at different $\Delta s_{\min }$ values, based on analyzing 500 nanowire devices before post processing. It can be seen that the number of qualified target nanowire candidates within each device decreases significantly as $\Delta s_{\text {min }}$ value increases. 
Table 4.3: Effect of $\Delta s_{\text {min }}$ value on failure rate of $N W$ severing.

\begin{tabular}{cccccc}
\hline \hline$\Delta s_{\min }(\mu \mathrm{m})$ & 5 & 3 & 2 & 1 & 0.5 \\
\hline fail $(\%)$ & 0 & 0 & 1 & 4 & 12 \\
\hline
\end{tabular}

Table 4.3 summarizes the nanomanipulation failure rate for different $\Delta s_{\min }$ values. A higher $\Delta s_{\min }$ value means the target nanowire is well separated from other neighboring nanowires resulting in a high success rate (e.g., $0 \%$ failure rate for $\Delta s_{\text {min }}=5 \mu \mathrm{m}$ and $\Delta s_{\text {min }}=3$ $\mu \mathrm{m}) . \Delta s_{\min }$ was selected in our experiments to be $1 \mu \mathrm{m}$ for a compromise between the nanomanipulation failure rate $(4 \%)$ and the number of qualified target nanowire candidates (i.e., 7 nanowires satisfying the $\Delta s_{\min }$ criterion and qualified for final selection based on desired nanowire diameter specification, $d_{r}$ ).

\subsubsection{Success Rate and Efficiency}

Figure 4.6 (a)(b) show a nano-FET device before and after robotic selective nanowire severing. Post processing permits specific selection of desired nanowire diameter and precise control of nanowire numbers. Figure 4.6 (c) shows an array of nano-FET devices all having a single nanowire bridging the source and drain electrodes. For a total of 500 trials, the success rate of the system was $95 \%$ (see Table 4.4), as compared to $48.3 \%$ for manual nanomanipulation. Failure in robotic nanowire severing resulted from accidentally fracturing the target nanowire $(2.2 \%)$ and the formation of undesired nanowire network (i.e., removed 'nano-junk' landed on top of the target nanowire) $(2.8 \%)$. Both nanowire networking and accidental target nanowire breakage were significantly lower compared to manual nanomanipulation as a direct result of precise execution of nanowire selection criteria and the high position performance of closed-loop position control. 


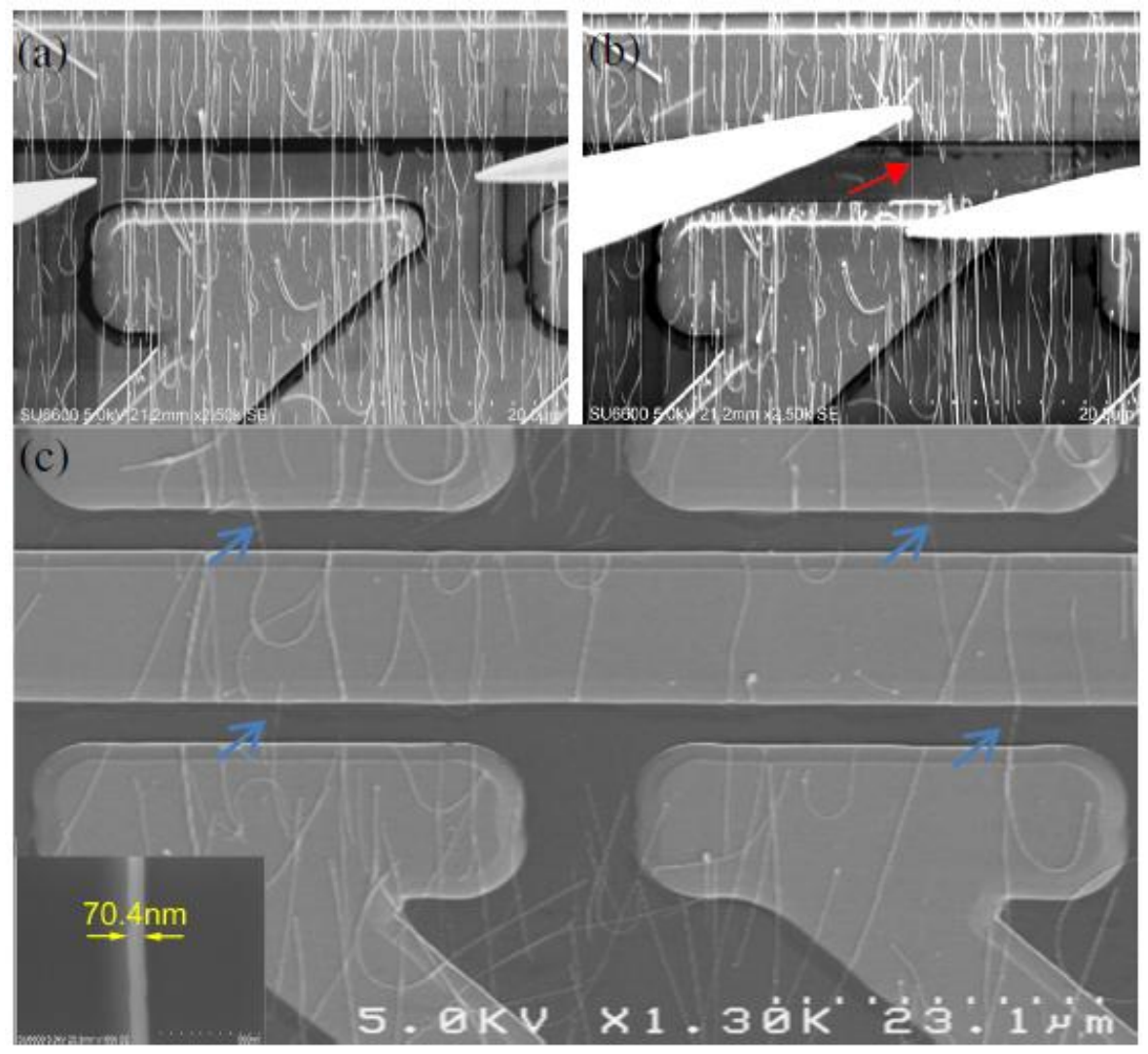

Figure 4.6: (a)(b) Before and after nanomanipulation removal of nanowires between source and drain electrodes. (c) An array of 4 single-nanowire devices (arrows: remaining desired nanowire). Inset shows a high-resolution SEM image of a target nanowire.

Table 4.4: Overall performance of nanorobotic manipulation.

\begin{tabular}{ccc}
\hline \hline failure cases $(\mathrm{n}=500)$ & target NW broken & networked \\
\hline 25 & 11 & 14 \\
$5 \%$ & $44 \%$ & $56 \%$ \\
\hline
\end{tabular}

The average time spent on post processing a nano-FET device was $\sim 1 \mathrm{~min}$. Compared to the average speed of manual nanomanipulation (10.3 min/device), automated robotic nanowire manipulation improved the post processing speed by $\sim 10$ times. Detailed break-up of the $1 \mathrm{~min}$ is: 9 seconds for nanowire detection and selection, 8 seconds for contact detection of the two 
nanoprobes, 22 seconds for position control of the two nanoprobes, and 20 seconds for probe cleaning. Typically, the tip of a nanoprobe requires cleaning after severing nanowires for 3-4 devices because of 'nanojunk' contaminant build-up. Nanoprobe cleaning is presently manually conducted by touching the nanoprobe tip gently over a piece of carbon tape used in SEM sample fixation, which takes approximately $1 \mathrm{~min}$. After processing about 20 devices, the nanoprobe tip was replaced because of significant contaminant build-up on the tip and/or because the probe tip became too thick for precision operation. Since replacing a nanoprobe can take minutes, we are presently pursuing approaches for in situ nanoprobe/tool exchange/replacement.

\subsubsection{Device Testing}

Since nanowire removal is a destructive process, care must be taken to prevent damage to the oxide layer on the substrate. Severing occurs along the edges of the electrodes and damage to the oxide layer will destroy the structure of the device by exposing the silicon substrate, effectively shorting the gate to the source/drain electrodes. In addition, during microfabrication, the nanowires on the device may not have ohmic contact with the two electrodes. Therefore, individual testing of each device to differentiate working biosensors from non-working biosensors is required.

Electrical characterization was performed to obtain device I-V characteristics, and to verify Ohmic nanowire-electrode contact, oxide layer damage, and proper n-type FET electrical characteristics, as shown in Figure 4.7. Electrical testing confirms that all devices exhibited stable signals. For single-nanowire devices, this indicates that each bridging nanowire in contact with the electrodes is conductive. Although it is difficult to determine if this is true for multinanowire devices, based on the results for single-nanowire devices, we trust that the overwhelming majority of bridging nanowires in multi-nanowire devices are also conductive. 


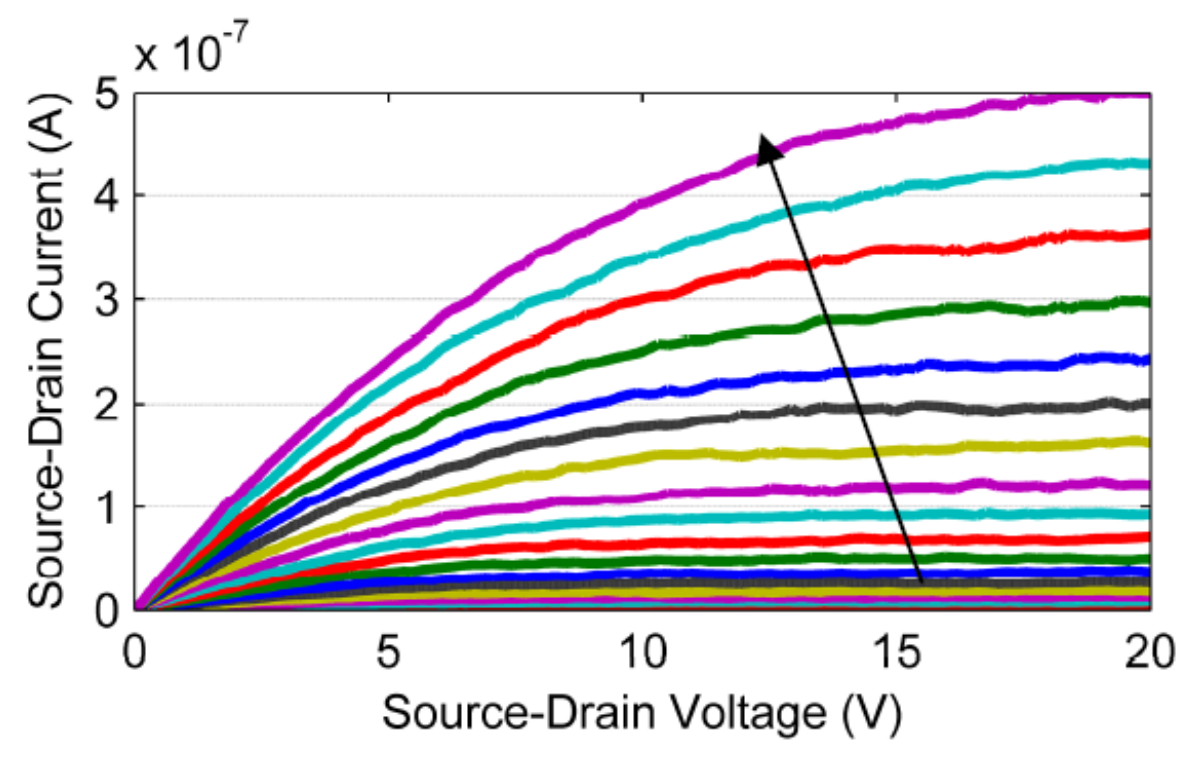

Figure 4.7: Current-voltage characteristics of a post-processed nanowire FET device $\left(\mathrm{V}_{\text {sd }}=\right.$ 0 to $20 \mathrm{~V}, \mathrm{~V}_{\text {backgate }}=-25$ to $+25 \mathrm{~V}$, increasing $\mathrm{V}_{\text {backgate }}$ indicated by arrow).

Devices were examined using high resolution SEM imaging for damage caused by the nanowire removal process. No visible defects of the substrate were visible from nanowire removal, indicating that contact detection successfully prevents this. Electrodes also showed no new defects after the nanowire removal process.

Testing of 10 single-nanowire transistor devices $(\mathrm{Vsd}=10 \mathrm{mV}, \mathrm{Vg}=0 \mathrm{~V})$ fabricated using the same batch of nanowires $\left(10^{19}\right.$ atoms $\left./ \mathrm{cm}^{3}\right)$ exhibited an average conductance of 1.06E$7 \mathrm{~S}(\sigma=4.79 \mathrm{E}-8)$. Variation in device conductance may be attributed to slight differences in nanowire properties (diameter, doping concentration, imperfections) and small differences in the contacting electrode lengths between devices Automated in-situ electrical testing through contact detection demonstrates its ability to detect working devices by verifying Ohmic contact making it an efficient validation tool for testing nano-FET device conductivity. 


\subsubsection{Varying Number and Diameter of Nanowires}

Automated nanomanipulation was used to post process nano-FET devices for controlling the number and diameter of bridging nanowires. Nanowire devices were then functionalized with anti-human IgG for hIgG protein detection experiments. Nano-FET source-drain currents were measured as a function of increasing concentrations of hIgG protein $\left(V_{s d}=0.01 \mathrm{~V}, V_{\text {gate }}=-1 \mathrm{~V}\right)$. Figure 4.8 shows a typical current-time response for $7 \mathrm{Si}$-nanowire biosensors exposed to increasing concentrations of hIgG. From these data, we determine the device sensitivity, defined as $\left(I-I_{0}\right)=I_{0}$.

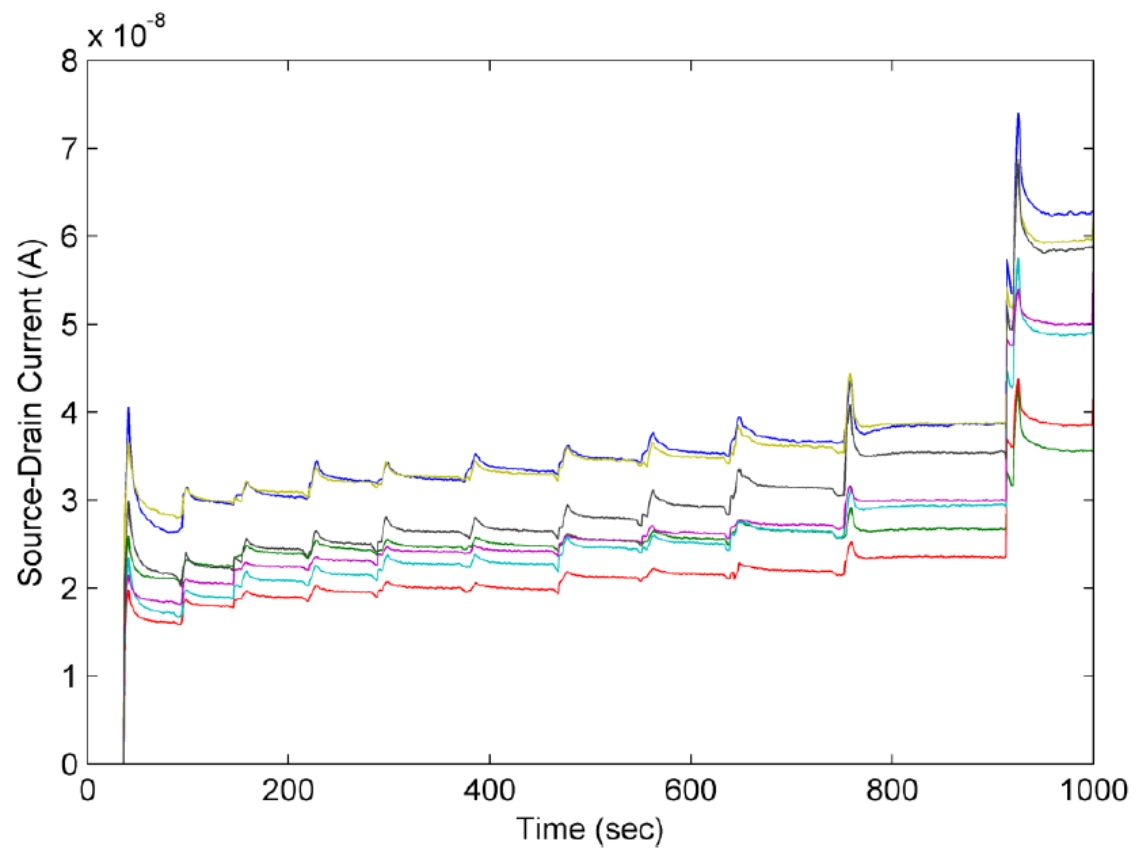

Figure 4.8: A typical current-time response for $7 \mathrm{Si}$-nanowire FET biosensors exposed to increasing concentrations of hIgG in $0.1 \times$ PBS buffer (from $10 \mathrm{fg} / \mathrm{mL}$ to $10 \mu \mathrm{L}$ ).

\subsubsection{Effect of Nanowire Number}

Previous studies have shown that the number of bridging nanowires incorporated into a device may be an important parameter in determining sensitivity [21, 75]. Zhang et al. [21] 
demonstrated that multiple $\mathrm{In}_{2} \mathrm{O}_{3}$ nanowire FET devices were more sensitive than single nanowire devices for gaseous chemical sensing due to the formation of nanowire network junctions. In contrast, Gruner et al. [75] further showed that individual carbon nanotube (CNT) FET biosensors, as compared to CNT network devices, exhibited higher sensitivity. Since these sensing systems differ from nano-FET biosensors in terms of sensing environment, molecule of interest and/or nanostructure composition and type, we investigate the dependence of nanowire number in nanowire-based biosensing.

Twelve devices with exactly 1,4 , or 7 bridging nanowires $(n=4$ for each group, $80-100$ $\mathrm{nm}$ diameter, nanowire doping density $=10^{19}$ atoms $\cdot \mathrm{cm}^{-3}$ ) were fabricated and post processed. Devices are tested against increasing concentrations of hIgG, as shown in Figure 4.9 (a)(b). All devices exhibited linear relationships between source-drain current and $\log [\mathrm{hIgG}]$. Device sensitivity was found to be maximum for single nanowire devices ( 0.05 per decade; $n=4)$ and decreased in sensitivity for increasing number of nanowires $(0.031$ per decade for 4 nanowire device and 0.009 per decade for 7 nanowire device, which represents a $\sim 38 \%$ and $\sim 81 \%$ decrease in sensitivity, respectively; $\mathrm{n}=4$ for both cases), which indicate that single nanowire devices yield the highest sensitivity. The significant difference in sensitivity also indicates that nano-FET devices having the same number of bridging nanowires through controlled nanomanipulation exhibit less sensitivity variation compared to devices with poorly controlled nanowire number. 

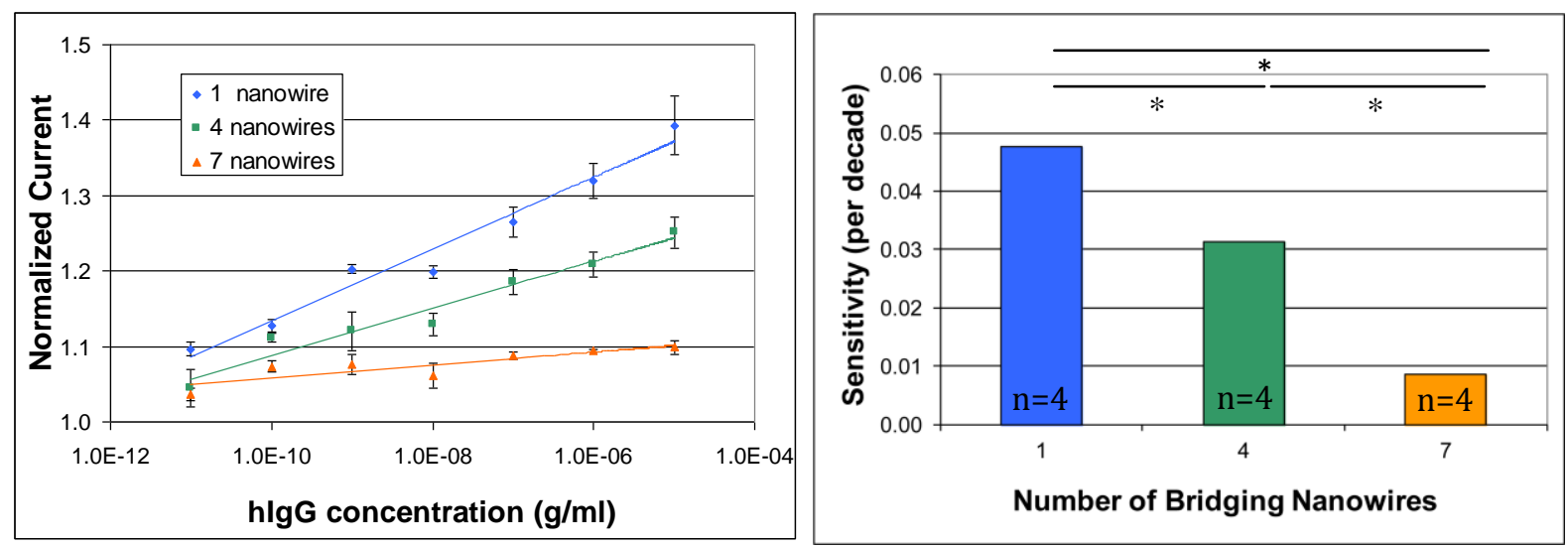

Figure 4.9: Effect of nanowire number on device sensitivity and variability. (a) Normalized current as a function of protein concentration for devices with 1,4 and 7 nanowires $(n=4$ for each group; nanowire diameter $80-100 \mathrm{~nm}$; nanowire doping density $=10^{19}$ atoms $^{\cdot} \mathrm{cm}^{-}$ ${ }^{3}$ ). (b) Sensitivity as a function of the number of bridging nanowires ( $n=4$ for each group). Decreasing sensitivity was observed for higher numbers of nanowires. $(* \mathbf{p}<0.05)$

Since our fabrication process eliminates the presence of nanowire networks, we can attribute this observation to the depletion of hIgG molecules in solution. For a single nanowire device, which experiences an initial source-drain current of $\mathrm{I}_{0(1 \mathrm{nw})}$, introducing the analyte solution results in the binding of $\mathrm{x}_{1 \mathrm{nw}}$ molecules onto the nanowire surface thereby producing a change in current $\left(\mathrm{I}-\mathrm{I}_{0}\right)_{1 \mathrm{nw}} / \mathrm{I}_{0(1 \mathrm{nw})}$. Similarly, for multi-nanowire devices, with $n$ nanowires connected in parallel and an initial source-drain current of $\mathrm{I}_{0(\mathrm{n} \text { nanowires })}=\mathrm{n} \times \mathrm{I}_{0(1 \mathrm{nw})}$, if we assume no appreciable depletion of analyte molecules from solution, then a total current change of (I$\left.\mathrm{I}_{0}\right)_{(\mathrm{n} \text { nanowires })}=n \times\left(\mathrm{I}-\mathrm{I}_{0}\right)_{1 \mathrm{nw}}$ would be expected. Under this assumption, the sensitivity of a nanoFET sensor with $n$ parallel nanowires is then $\left(\mathrm{I}-\mathrm{I}_{0}\right)_{(\mathrm{n} \text { nanowires })} / \mathrm{I}_{0(\mathrm{n} \text { nanowires })}=n\left(\mathrm{I}-\mathrm{I}_{0}\right)_{1 \mathrm{nw}} / n \mathrm{I}_{0(1 \mathrm{nw})}=(\mathrm{I}-$ $\left.\mathrm{I}_{0}\right)_{1 \mathrm{nw}} / \mathrm{I}_{0(1 \mathrm{nw})}$, which is equivalent to that of a single nanowire device. However, for low concentrations of analyte, a finite number of analyte molecules are located in the immediate vicinity around the nano-FET device. Therefore the effect of analyte depletion from the 
surrounding solution becomes significant [76, 77] and effectively decreases the number of nanowire-analyte binding events experienced per nanowire. Thus, for a multi-nanowire FET device in which multiple closely spaced nanowires compete for the binding of free analyte molecules, the number of nanowire-analyte interactions for each nanowire is expected to decrease for increasing numbers of nanowires such that $\mathrm{x}_{(\mathrm{n} \text { nanowires })}<\mathrm{x}_{1 \mathrm{nw}}$ resulting in a smaller current change for each nanowire. Therefore, a lower sensitivity is expected for nano-FET devices with multiple nanowires (i.e., $\left.\left(\mathrm{I}-\mathrm{I}_{0}\right)_{(\mathrm{n} \text { nanowires })} / \mathrm{I}_{0(\mathrm{n} \text { nanowires })}<\left(\mathrm{I}-\mathrm{I}_{0}\right)_{1 \mathrm{nw}} / \mathrm{I}_{0(1 \mathrm{nw})}\right)$. Further studies are needed to conclude analyte depletion as the cause for our observations

\subsubsection{Effect of Nanowire Diameter}

Three groups of single-nanowire biosensors were fabricated with nanowire diameters of 60-80 nm, 81-100 nm, and 101-110 nm (n=6 for each case) with nanowire length of $5 \mu m$. We investigate the effect of nanowire diameter on nano-FET sensitivity using these three groups. While sensitivity dependence on nanowire diameter has been demonstrated in several nano-FET gas[78] and $\mathrm{pH}$ sensors[22, 23], few experimental studies have examined this dependence in biosensing applications. Sensors were tested with increasing concentrations of hIgG to determine device sensitivity. Device sensitivity consistently increased as nanowire diameter decreased (0.192 per decade to 0.116 per decade, $60.6 \%$ sensitivity difference; $n=6$ ), as shown in Figure 4.10. This trend is in agreement with experimental $\mathrm{pH}$ measurements made by Stern et al. [79] and shows that thinner wires exhibit higher sensitivity which is in agreement with modeling predictions reported by Nair et al. [70]. 


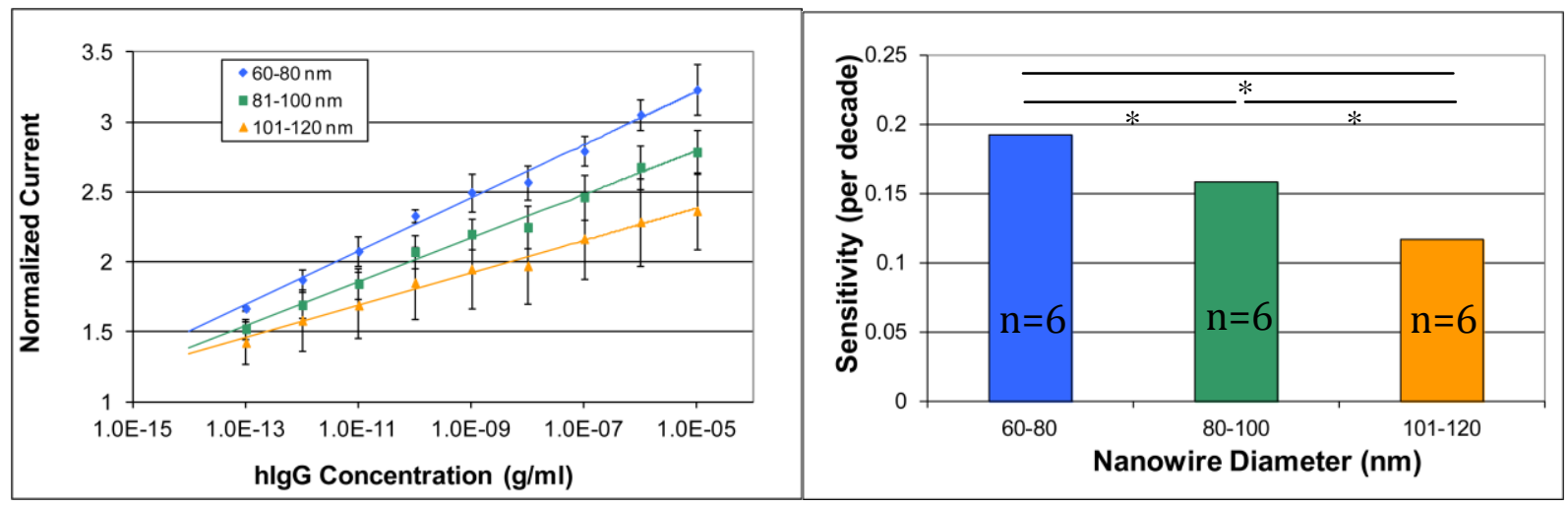

Figure 4.10: Effect of nanowire diameter on device sensitivity. Single-nanowire devices with mean diameters of 60-80 $\mathrm{nm}, 81-100 \mathrm{~nm}$, and 101-120 $\mathrm{nm}$ were characterized. (a) Normalized current as a function of protein concentration for single-nanowire devices with different nanowire diameters ( $\mathrm{n}=6$ for each group, doping concentration of $10^{17}$ ). Smaller nanowire diameters produced more sensitive nanowire FET biosensors. ( $\mathrm{n}=6$ for each group; $* \mathbf{p}<0.05)$.

The significant sensitivity difference among these three groups exemplifies the importance in controlling nanowire diameter to reduce device to device variability. Rather than obtaining the general performance from a batch of biosensors, the proposed nanomanipulation techniques enables the option to define the performance of each individual biosensor by choosing the appropriate size nanowire.

\subsection{Conclusion}

We presented a technique for batch microfabrication and serial post processing of nanowire devices. The batch microfabrication process is wafer-scale but results in bridging nanowires of varying nanowire number and diameter. Nanorobotic selective nanowire removal, despite being a serial process, permits precision control of the number and diameter of nanowires. Experimental results demonstrate that the nanorobotic system has a nano-FET device 
post processing success rate of $95 \%$ (vs. $48.3 \%$ for manual nanomanipulation) and has a speed of $1 \mathrm{~min} /$ device (vs. $10.3 \mathrm{~min} /$ device). The significant improvement in both success rate and efficiency resulted from automated operation that enabled specific nanowire selection and precision position control.

Our results demonstrate nanowire removal as an effective tool for reducing device variability by producing devices with desired nanowire number and diameter. We constructed three groups of nano-FET devices with differing number of bridging nanowires. Single nanowire devices were shown to be generally more sensitive than multi-nanowire devices. However, we hypothesize that the decrease in sensitivity for multi-nanowire devices is due to competitive binding and depletion of analyte for devices whose nanowires are in close proximity with each other. This suggests that spacing of nanowires in multi-nanowire devices also play a significant role in device performance. Further experimentation is needed to investigate this phenomenon. Nano-FET biosensors with different nanowire diameters were also constructed and we experimentally demonstrate the effect of nanowire diameter on nano-FET biosensor sensitivity. We show that smaller diameter nanowires exhibit higher sensitivities than larger diameter nanowires. In summary, our nanomanipulation post-processing method is capable of selectively controlling nanowire number and diameter allowing for nano-FET sensor optimization, both in terms of improving device sensitivity and minimizing device variability. 


\section{Chapter 5 \\ Conclusion and Future Work}

\subsection{Conclusion}

The first objective was to develop a computer-vision based contact detection algorithm for the reliable and repeatable approach of the end-effector to the target surface. In achieving this, we developed a contact detection algorithm that is capable of positioning an end-effector in contact with a target surface, achieving accuracies down to $20 \mathrm{~nm}$. The end-effector is first brought into alignment within the depth of focus, followed by detection of probe-substrate contact by monitoring an abrupt shift in motion. Our contact detection algorithm minimizes endeffector damage by detecting contact swiftly, thereby minimizing the deflection distance of the probe tip and minimizing the effects of permanent deformation. This method does not require any additional sensors or devices and is versatile for use in many nanomanipulation processes.

We then apply this contact detection algorithm for the automated post-processing of nano-FET biosensors with improved nanowire control and demonstrate its ability to reduce device-to-device variability, which translates to minimized performance variability. In doing so, we developed a new fabrication method that allowed careful control over several device parameters, including the number of nanowires incorporated into each device and the nanowire diameter, which effectively reduces device variability. Fabricated nano-FET sensors are then used to study the influence of nanowire parameters on device sensitivity and the results suggest that each of these parameters are critical in determining nano-FET biosensor performance. Specifically, we found that single-nanowire devices exhibit improved biomolecular sensitivity when compared to multi-nanowire devices, demonstrating the importance in selecting nanowire

number to reduce device variability. Smaller nanowire diameters also exhibit increased 
sensitivity and our nanowire removal process is capable of reducing variability by selectively choosing desired nanowire diameter resulting in less variability within a group of nano-FET sensors. Our results also suggest that the spacing between individual nano-FET sensors in a sensor array can influence device sensitivity which needs further investigation.

Contact detection is an essential tool in automated nanomanipulation. Without the need for additional vision sensors or equipment, and end-effector can be positioned within close proximity to the substrate repeatedly. Within this research, contact detection is demonstrated as a valuable tool in nanomanipulation for the large-scale fabrication of nanoscale devices and is capable in reducing device-to-device variability, thereby paving the way to continue bridging the gap between bottoms-up and top-down fabrication methods.

\subsection{Future Work}

\subsubsection{Contact Detection using Nanogrippers}

While automated grasping of microscale objects has been achieved, automated grasping of nanoscale objects has demonstrated much higher difficulty as physical phenomena such as adhesion forces are much more apparent at the nanoscale. Implementing contact detection within these applications can prove to be beneficial to automated nanomanipulation to form novel nanostructures.

\subsubsection{Investigate the Effects of Nanowire Spacing in Biosensing}

We have demonstrated through our results that nano-FET biosensors comprised of fewer nanowires exhibit increased device sensitivity. We hypothesized that this was due to noncompetitive analyte binding and depletion of single nanowire devices versus multi-nanowire 
devices. Sensors with multi-nanowires and controlled distances between them need to be fabricated and tested to determine the effects of spacing on device sensitivity.

\subsubsection{Expand on Automated Nanomanipulation Applications}

We now have a contact detection algorithm suitable for automated nanomanipulation. Extending our capability to be able to fabricate other types of functional devices may prove noteworthy in applications as; the construction of photonic crystals, handling biological cells, and the construction of complex micro/nano systems. 


\section{References}

1. Nguyen, C.V. and et al., Carbon nanotube tip probes: stability and lateral resolution in scanning probe microscopy and application to surface science in semiconductors. Nanotechnology, 2001. 12(3): p. 363.

2. Law, J.B.K. and J.T.L. Thong, Simple fabrication of a $\mathrm{ZnO}$ nanowire photodetector with a fast photoresponse time. Vol. 88. 2006: AIP. 133114.

3. $\quad$ Fan, Z., et al., ZnO nanowire field-effect transistor and oxygen sensing property. Applied Physics Letters, 2004. 85(24): p. 5923-5925.

4. Zhang, D., et al., Detection of NO2 down to ppb Levels Using Individual and Multiple In2O3 Nanowire Devices. Nano Letters, 2004. 4(10): p. 1919-1924.

5. Zhang, Z., et al., Synthesis of Ba-doped $\mathrm{CeO} 2$ nanowires and their application as humidity sensors. Nanotechnology, 2007. 18(46): p. 465504.

6. Huang, B., et al., Adsorption of Gas Molecules on Graphene Nanoribbons and Its Implication for Nanoscale Molecule Sensor. The Journal of Physical Chemistry C, 2008. 112(35): p. 13442-13446.

7. Cui, Y., et al., Nanowire Nanosensors for Highly Sensitive and Selective Detection of Biological and Chemical Species. Science, 2001. 293(5533): p. 1289-1292.

8. Hahm, J.-i. and C.M. Lieber, Direct Ultrasensitive Electrical Detection of DNA and DNA Sequence Variations Using Nanowire Nanosensors. Nano Letters, 2003. 4(1): p. 51-54.

9. $\quad$ Li, Z., et al., Sequence-Specific Label-Free DNA Sensors Based on Silicon Nanowires. Nano Letters, 2004. 4(2): p. 245-247.

10. Zhang, G.-J., et al., Silicon nanowire biosensor for highly sensitive and rapid detection of Dengue virus. Sensors and Actuators B: Chemical, 2010. 146(1): p. 138-144.

11. So, H.-M., et al., Single-Walled Carbon Nanotube Biosensors Using Aptamers as Molecular Recognition Elements. Journal of the American Chemical Society, 2005. 127(34): p. 11906-11907.

12. Gao, Z., et al., Silicon Nanowire Arrays for Label-Free Detection of DNA. Analytical Chemistry, 2007. 79(9): p. 3291-3297.

13. Elfstrom, N., A.E. Karlstrom, and J. Linnros, Silicon nanoribbons for electrical detection of biomolecules. Nano Letters, 2008. 8(3): p. 945-949.

14. Stern, E., et al., Label-free immunodetection with CMOS-compatible semiconducting nanowires. Nature, 2007. 445(7127): p. 519-522. 
15. Rao, S.G., et al., Nanotube electronics: Large-scale assembly of carbon nanotubes. Nature, 2003. 425(6953): p. 36-37.

16. Fan, Z., et al., Wafer-Scale Assembly of Highly Ordered Semiconductor Nanowire Arrays by Contact Printing. Nano Letters, 2007. 8(1): p. 20-25.

17. Vijayaraghavan, A., et al., Ultra-Large-Scale Directed Assembly of Single-Walled Carbon Nanotube Devices. Nano Letters, 2007. 7(6): p. 1556-1560.

18. Ford, A.C., et al., Diameter-dependent electron mobility of InAs nanowires. Nano Lett, 2009. 9(1): p. 360-5.

19. Black, C.T., Self-aligned self assembly of multi-nanowire silicon field effect transistors. Applied Physics Letters, 2005. 87(16): p. 163116-163116-3.

20. Fan, Z. and J.G. Lu, Chemical Sensing with ZnO Nanowire Field-Effect Transistor. IEEE Transactions on Nanotechnology, 2006. 5(4): p. 393-396.

21. Zhang, D., et al., Detection of NO2 down to ppb Levels Using Individual and Multiple In2O3 Nanowire Devices. Nano Lett, 2004. 4(10): p. 1919-1924.

22. Stern, E., et al., Label-free immunodetection with CMOS-compatible semiconducting nanowires. Nature, 2007. 445(7127): p. 519-22.

23. Elfstrom, N., et al., Surface charge sensitivity of silicon nanowires: size dependence. Nano Lett, 2007. 7(9): p. 2608-12.

24. Elfstrom, N., A.E. Karlstrom, and J. Linnros, Silicon nanoribbons for electrical detection of biomolecules. Nano Lett, 2008. 8(3): p. 945-9.

25. Li, Z., et al., Silicon nanowires for sequence-specific DNA sensing: device fabrication and simulation. Applied Physics A: Materials Science \& Processing, 2005. 80(6): p. 1257-1263.

26. Cagliani, A., et al., Manipulation and in situ transmission electron microscope characterization of sub-100 $\mathrm{nm}$ nanostructures using a microfabricated nanogripper. Journal of Micromechanics and Microengineering, 2010. 20(3): p. 035009.

27. Dong, L., F. Arai, and T. Fukuda, Destructive constructions of nanostructures with carbon nanotubes through nanorobotic manipulation. Mechatronics, IEEE/ASME Transactions on, 2004. 9(2): p. 350-357.

28. Fukuda, T., F. Arai, and L. Dong, Assembly of nanodevices with carbon nanotubes through nanorobotic manipulations. Proceedings of the IEEE, 2003. 91(11): p. 18031818.

29. Fukuda, T., et al., Nanofabrication, nanoinstrumentation and nanoassembly by nanorobotic manipulation. International Journal of Robotics Research, 2009. 28(4): p. 537-547. 
30. Mølhave, K., et al., Pick-and-place nanomanipulation using microfabricated grippers. Nanotechnology, 2006. 17(10): p. 2434.

31. Sharma, H.D., T. Pal, and C. Shekhar, Characterization of microcantilever spring and force sensor using nanomanipulators. Thin Solid Films, 2010. 519(3): p. 1040-1043.

32. Aoki, K., et al., Microassembly of semiconductor three-dimensional photonic crystals. Nat Mater, 2003. 2(2): p. 117-121.

33. Eichhorn, V., et al., Depth-detection methods for microgripper based CNT manipulation in a scanning electron microscope. Journal of Micro - Nano Mechatronics, 2008. 4(1): p. 27-36.

34. Kasaya, T., et al. Micro-object handling under SEM by vision-based automatic control. in Microrobotics and Micromanipulation. 1998. Boston, MA, USA: SPIE.

35. Burkle, A. and F. Schmoeckel, Quantitative measuring system of a flexible microrobotbased microassembly station. 4th Seminar on Quantitative Microscope, Semmering, Austria, 2000: p. 191-198.

36. Cvetanovic, A., et al., Design of a novel visual and control system for the prevention of the collision during the micro handling in a SEM chamber. Microelectronic Engineering, 2010. 87(2): p. 139-143.

37. Kim, D.-H. and et al., A superelastic alloy microgripper with embedded electromagnetic actuators and piezoelectric force sensors: a numerical and experimental study. Smart Materials and Structures, 2005. 14(6): p. 1265.

38. Trinh Chu, D., et al., Electrothermal Microgripper With Large Jaw Displacement and Integrated Force Sensors. Microelectromechanical Systems, Journal of, 2008. 17(6): p. 1546-1555.

39. Arai, F., et al. Novel touch sensor with piezoelectric thin film for microbial separation. in Robotics and Automation, 2003. Proceedings. ICRA '03. IEEE International Conference on. 2003.

40. Motoo, K., et al., High sensitive touch sensor with piezoelectric thin film for pipetting works under microscope. Sensors and Actuators A: Physical, 2006. 126(1): p. 1-6.

41. Xinyu Liu, et al., Nanonewton Force Sensing and Control in Microrobotic Cell Manipulation. The International Journal of Robotics Research, 2009. 28(8): p. 10651076.

42. Ribeiro, E. and M. Shah, Computer Vision for Nanoscale Imaging. Machine Vision and Applications, 2006. 17(3): p. 147-162.

43. Abe, K., et al. Three-dimensional measurement by tilting and moving objective lens in CD-SEM(III). in Metrology, Inspection, and Process Control for Microlithography XIX. 2005. San Jose, CA, USA: SPIE. 
44. Fuller, K., et al., Automated three-dimensional characterization of osteoclastic resorption lacunae by stereoscopic scanning electron microscopy. Journal of Bone and Mineral Research, 1994. 9(1): p. 17-23.

45. Jähnisch, M. and S. Fatikow, 3-D Vision Feedback for Nanohandling Monitoring in a Scanning Electron Microscope. Int. J. Optomechatronics, 2007. 1(1): p. 4 - 26.

46. Ohba, K., et al., Microscopic vision system with all-in-focus and depth images. Machine Vision and Applications, 2003. 15(2): p. 55-62.

47. Yu Sun, S.D., Bradley J. Nelson,, Autofocusing in computer microscopy: Selecting the optimal focus algorithm. Microscopy Research and Technique, 2004. 65(3): p. 139-149.

48. Eichhorn, V., et al. NanoLab: A nanorobotic system for automated pick-and-place handling and characterization of CNTs. in Robotics and Automation, 2009. ICRA '09. IEEE International Conference on. 2009.

49. Huang, Y., et al., Directed Assembly of One-Dimensional Nanostructures into Functional Networks. Science, 2001. 291(5504): p. 630-633.

50. $\quad$ Fan, Z., et al., Wafer-scale assembly of highly ordered semiconductor nanowire arrays by contact printing. Nano Lett, 2008. 8(1): p. 20-5.

51. $\mathrm{Yu}, \mathrm{G}$, et al., Nanomaterial-incorporated blown bubble film for large-area aligned nanostructures. J. Mater. Chem., 2008. 18: p. 728-734.

52. Lee, M., et al., Linker-free directed assembly of high-performance integrated devices based on nanotubes and nanowires. Nat Nanotechnol, 2006. 1(1): p. 66-71.

53. Vijayaraghavan, A., et al., Ultra-large-scale directed assembly of single-walled carbon nanotube devices. Nano Lett, 2007. 7(6): p. 1556-60.

54. Chang, M., et al., Assembly of nanostructures by using a mechanical nanomanipulator. Key Eng. Mat., 2011. 450: p. 263-266.

55. Donald, B.R., C.G. Levey, and I. Paprotny, Planar Microassembly by Parallel Actuation of MEMS Microrobots. Microelectromechanical Systems, Journal of, 2008. 17(4): p. 789808.

56. Kumar, R.T.R. and et al., Nanobits: customizable scanning probe tips. Nanotechnology, 2009. 20(39): p. 395703.

57. $\quad \mathrm{Ru}, \mathrm{C}$., et al., Automated Four-Point Probe Measurement of Nanowires inside a Scanning Electron Microscope. Nanotechnology, IEEE Transactions on, 2010(99).

58. Zhang, H., et al., Microassembly Fabrication of Tissue Engineering Scaffolds With Customized Design. Automation Science and Engineering, IEEE Transactions on, 2008. 5(3): p. 446-456. 
59. Dong, L., et al., Towards nanotube linear servomotors. Automation Science and Engineering, IEEE Transactions on, 2006. 3(3): p. 228-235.

60. Peng, Y., T. Cullis, and B. Inkson, Bottom-up Nanoconstruction by the Welding of Individual Metallic Nanoobjects Using Nanoscale Solder. Nano Letters, 2008. 9(1): p. 91-96.

61. Peng, Y., T. Cullis, and B. Inkson, Accurate electrical testing of individual gold nanowires by in situ scanning electron microscope nanomanipulators. Applied Physics Letters, 2008. 93(18): p. 183112.

62. Yong Ju, Y. and et al., Manipulation of freestanding Au nanogears using an atomic force microscope. Nanotechnology, 2007. 18(50): p. 505304.

63. Yu, M., et al., Three-dimensional manipulation of carbon nanotubes under a scanning electron microscope. Nanotechnology, 1999. 10(3): p. 244.

64. Liu, X., et al., Nanonewton force-controlled manipulation of biological cells using a monolithic MEMS microgripper with two-axis force feedback. Journal of Micromechanics and Microengineering, 2008. 18(5): p. 055013.

65. Wang, W.H., X.Y. Liu, and Y. Sun, Contact detection in microrobotic manipulation. International Journal of Robotics Research, 2007. 26(8): p. 821-828.

66. Kratochvil, B.E., Lixin Dong, and B.J. Nelson, Real-time Rigid-body Visual Tracking in a Scanning Electron Microscope. The International Journal of Robotics Research, 2009. 28(4): p. 498-511.

67. Reimer, L., Scanning electron microscopy: physics of image formation and microanalysis. 1998, Berlin: Springer

68. Gimzewski, J.K., ouml, and R. ller, Transition from the tunneling regime to point contact studied using scanning tunneling microscopy. Physical Review B, 1987. 36(2): p. 1284.

69. Zhou, W., R.P. Apkarian, and Z.L. Wang, Fundamentals of Scanning Electron Microscopy, in Scanning Microscopy for Nanotechnology: Techniques and Applications, W. Zhou and Z.L. Wang, Editors. 2007, Springer: New York. p. 1-40.

70. Nair, P.R. and M.A. Alam, Design Considerations of Silicon Nanowire Biosensors. Electron Devices, IEEE Transactions on, 2007. 54(12): p. 3400-3408.

71. Nair, P.R. and M.A. Alam, Screening-limited response of nanobiosensors. Nano Lett, 2008. 8(5): p. 1281-5.

72. Zhang, Y.L., et al., A load-lock compatible nanomanipulation system for scanning electron microscope. IEEE/ASME Trans. Mechatron., 2011. 
73. Ji, L. and H. Yan, Attractable snakes based on the greedy algorithm for contour extraction. Pattern Recognition, 2002. 35(4): p. 791-806.

74. Harmanson, G.T., Bioconjugate Techniques. 2nd ed. 2008: Elsevier Inc.

75. Gruner, G., Carbon nanotube transistors for biosensing applications. Anal Bioanal Chem, 2006. 384(2): p. 322-35.

76. Squires, T.M., R.J. Messinger, and S.R. Manalis, Making it stick: convection, reaction and diffusion in surface-based biosensors. Nat Biotechnol, 2008. 26(4): p. 417-26.

77. Sheehan, P.E. and L.J. Whitman, Detection limits for nanoscale biosensors. Nano Lett, 2005. 5(4): p. 803-7.

78. Fan, Z. and L.J. G., Chemical sensing with $\mathrm{ZnO}$ nanowire field-effect transistor. Nanotechnology, IEEE Transactions on, 2006. 5(4): p. 393-396.

79. Stern, E., et al., Importance of the Debye screening length on nanowire field effect transistor sensors. Nano Lett, 2007. 7(11): p. 3405-9. 